University of Texas at El Paso

\title{
DigitalCommons@UTEP
}

Open Access Theses \& Dissertations

2014-01-01

\section{Numerical Prediction Of Collection Efficiency Of A Personal Sampler Based On Cyclone Principle}

Antara Badhan

University of Texas at El Paso, abadhan@miners.utep.edu

Follow this and additional works at: https://digitalcommons.utep.edu/open_etd

Part of the Mechanical Engineering Commons

\section{Recommended Citation}

Badhan, Antara, "Numerical Prediction Of Collection Efficiency Of A Personal Sampler Based On Cyclone Principle" (2014). Open Access Theses \& Dissertations. 1203.

https://digitalcommons.utep.edu/open_etd/1203

This is brought to you for free and open access by DigitalCommons@UTEP. It has been accepted for inclusion in Open Access Theses \& Dissertations by an authorized administrator of DigitalCommons@UTEP. For more information, please contact lweber@utep.edu. 


\title{
NUMERICAL PREDICTION OF COLLECTION EFFICIENCY OF A PERSONAL SAMPLER BASED ON CYCLONE PRINCIPLE
}

\author{
ANTARA BADHAN \\ Department of Mechanical Engineering
}

APPROVED:

Shaolin Mao, Ph.D., Chair

Norman Love, Ph.D.

Grace Yan, Ph.D.

Charles H. Ambler, Ph.D.

Dean of the Graduate School 


\section{Copyright}

by

Antara Badhan

2014 


\section{Dedication}

To my father M. A. Rahman, my mother Nurzahan Begum \& my husband Mohammad Arif Ishtiaque Shuvo for their support and encouragement throughout my life. 


\title{
NUMERICAL PREDICTION OF COLLECTION EFFICIENCY OF A PERSONAL SAMPLER BASED ON CYCLONE PRINCIPLE
}

\author{
by \\ ANTARA BADHAN, Bachelor of Science in Civil Engineering
}

\section{THESIS}

Presented to the Faculty of the Graduate School of

The University of Texas at El Paso

in Partial Fulfillment

of the Requirements

for the Degree of

MASTER OF SCIENCE

Department of Mechanical Engineering

THE UNIVERSITY OF TEXAS AT EL PASO

August 2014 


\section{Acknowledgements}

It would never have been possible to complete my thesis without the excellent guidance of my committee members, encouragement from my friends and support from my family. I would like to express my deepest gratitude to my research supervisor, Dr. Shaolin Mao for his continuous support and encouragement. His expert guidance, valuable comments and useful critiques has been a great help for my research. I would also like to thank Dr. Love and Dr. Yan for being part of my thesis committee members. My completion of this project could not have been accomplished without the financial support of Department of Mechanical Engineering. The assistance provided by the Department of Mechanical Engineering is greatly appreciated and I am really grateful to the department for providing such an opportunity to do research in an excellent environment. I wish to thank to all the Mechanical Engineering Department staffs for their generous help and assistance. My grateful thanks to my fellow mate Luz Bugarin for her co-operation and technical support on this project. A special thank goes to all my friends from cSETR Computational Lab for their support and encouragement throughout my thesis. Finally, I

owe more than thanks to my family for being always by my side and for helping and encouraging me throughout my life. 


\begin{abstract}
A personal bio-aerosol sampler is a self-contained, operation flexible, high-efficient device for indoor air quality (IAQ) and health risk exposure monitoring and measurement. Bioaerosols such germ-laden viruses, microbial species, airborne microorganisms and volatile organic compounds (VOC) are sucked into the sampler and are deposited on the inner wall surface based on cyclone principal. The major concern with bio-aerosol samplers is the collection efficiency. In this study, we used computational fluid dynamics (CFD) tools to evaluate key design parameters, specifically the inlet tube angle and collection tube inner wall roughness. 3D incompressible turbulent flow was simulated using commercial software ANSYS FLUENT. Reynolds stress model (RSM) was used to investigate the turbulence effect with the following boundary conditions (velocity-inlet boundary condition at inlet, outflow boundary condition at outlet and no slip at walls). The numerical approach for air-aerosol interaction is based on an Eulerian-Lagrangian fluid dynamics framework, where the particles or droplets trajectories are computed in a Lagrangian method (discrete phase element) and then conjugate these particles to the continuous phase in the Eulerian frame. The variation of inlet angle affects the collection efficiency of the cyclone sampler. In addition, the flow characterizations with different velocity fluctuation profiles validate the continuous phase model. The development and evolution of the vortex core regions for velocities are obtained and evaluated in the simulation of the cyclonic flow.
\end{abstract}




\section{Table of Contents}

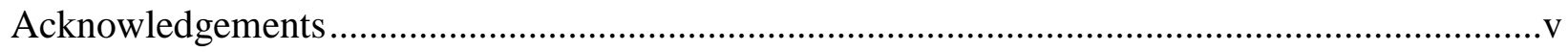

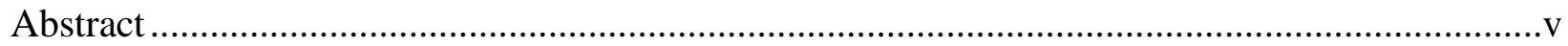

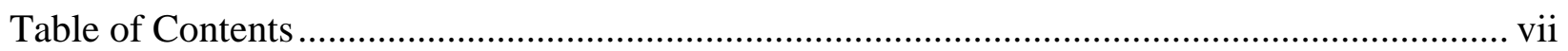

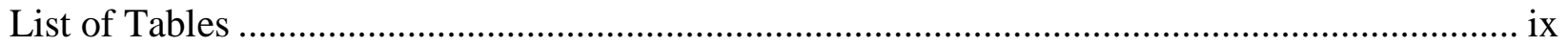

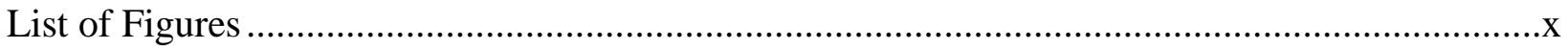

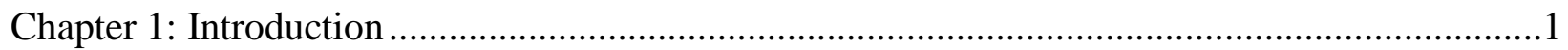

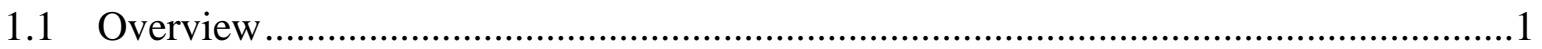

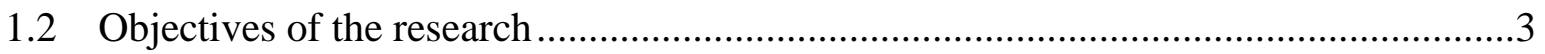

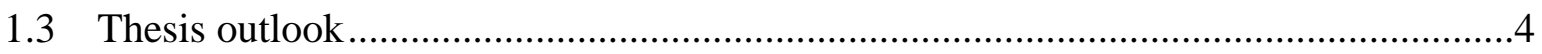

Chapter 2: Literature Review ..........................................................................................

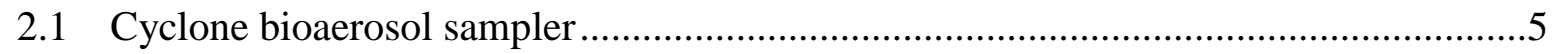

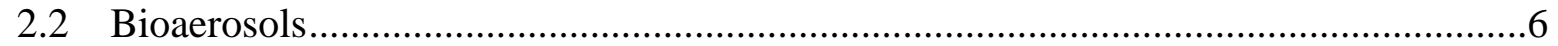

2.3 Bioaerosol sampler selection ..................................................................

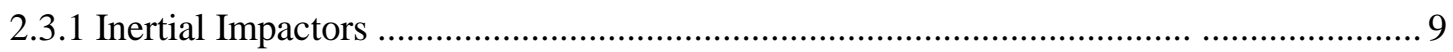

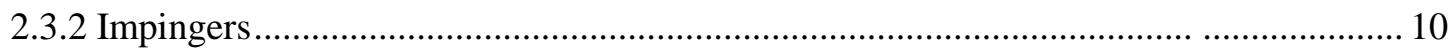

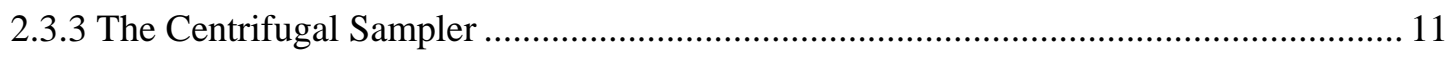

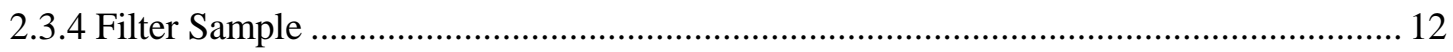

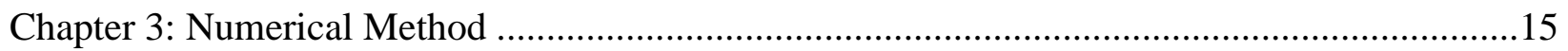

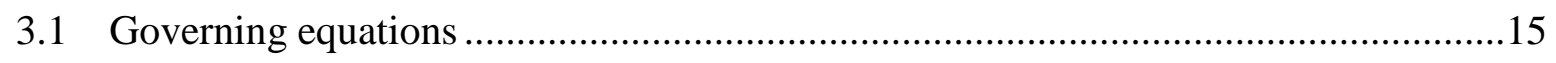

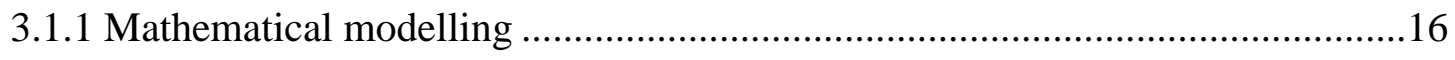

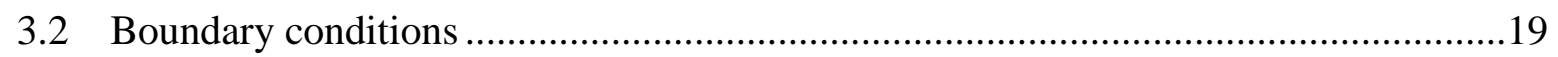

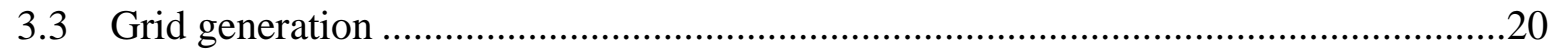

Chapter 4: Methodology of Cyclone Sampler Simulation....................................................24

4.1 Geometry of cyclone-based bioaerosol sampler ...................................................24

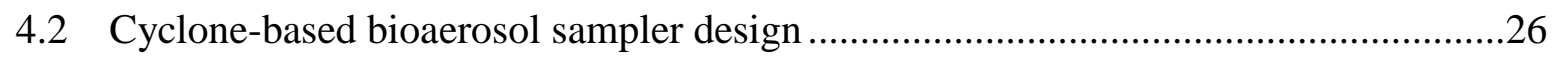

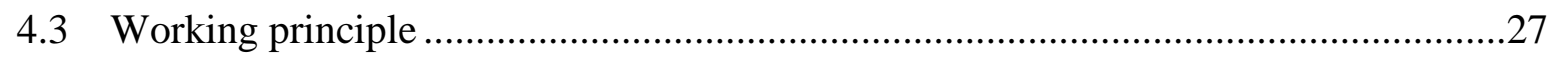

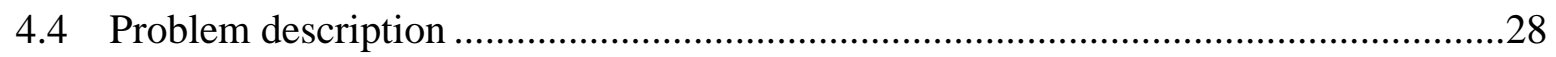

4.5 Calculation of collection efficiency for cyclone sampler .....................................31

4.6 Cyclone sapmler modeling in workbench..........................................................32 


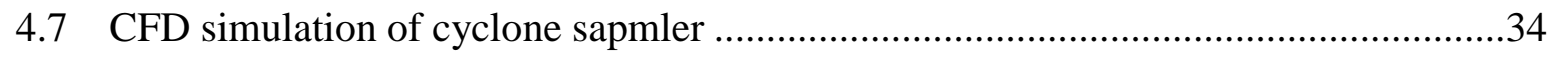

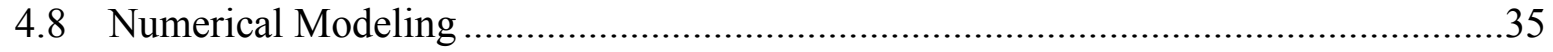

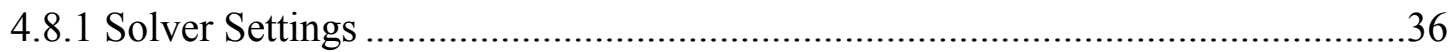

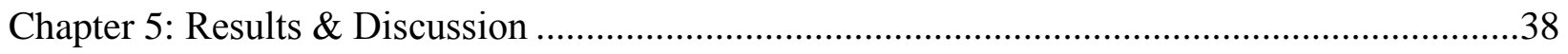

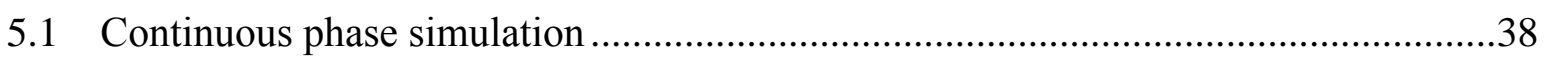

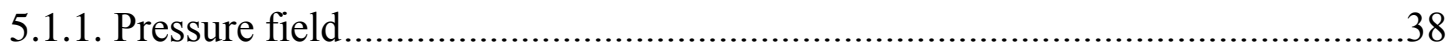

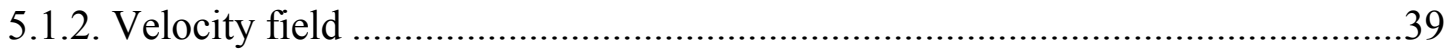

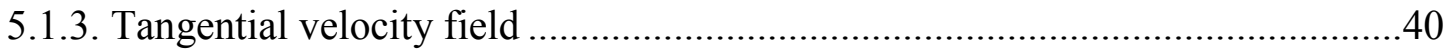

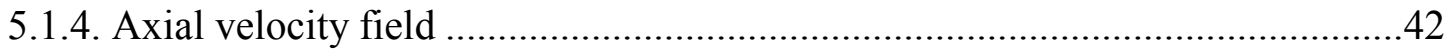

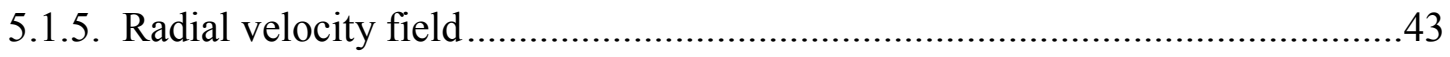

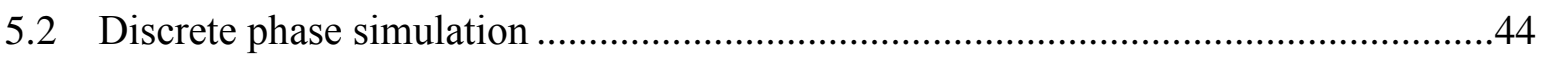

5.2.1. Particle trajectories for particle residence time..............................................44

5.2.2. Particle trajectories for particle velocity magnitude .....................................46

5.2.3. Particle trajectories for particle y velocity magnitude ...................................47

5.3 Streamline Pattern for Cyclone Sampler..............................................................48

5.4 Dimensionless velocity profiles measurement.................................................49

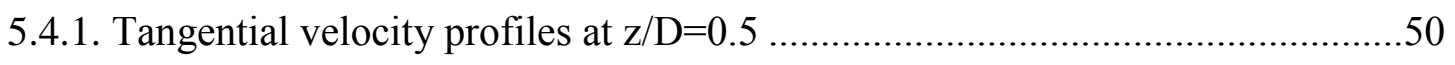

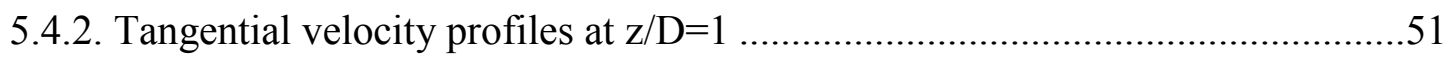

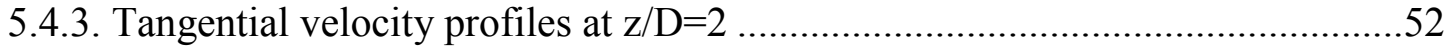

5.5 Velocity profile in vector plot for cyclone sampler ...........................................54

5.6 Flow pattern on different plane of cyclone sampler .........................................57

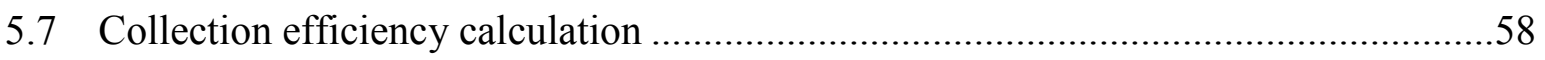

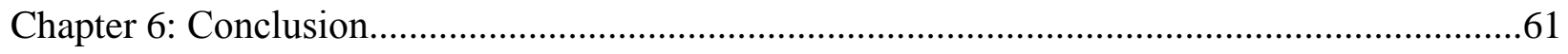

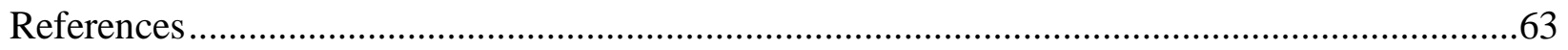

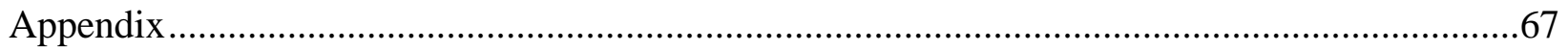

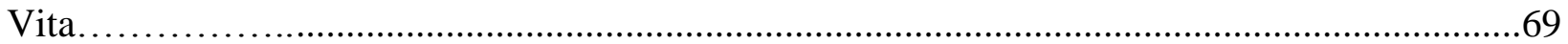




\section{List of Tables}

Table 2.2: Commonly used and commercially available bioaerosol samplers ........................... 9 Table 4.1: Geometrical dimensions of various microcentrifuge tubes used in bioaerosol samplers 25

Table 4.2: Geometric configuration of Cyclone studied for this project ................................. 29

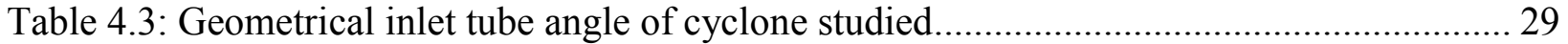

Table 4.4: Mesh sizes for four bioaerosol samplers used in this study................................... 32

Table 5.1: Collection efficiency data studied for this project ............................................... 59 


\section{List of Figures}

Figure 2.1: Portable bioaerosol sampler for bioaerosol collection ....................................... 6

Figure 2.2: Examples of different aerosol types from an electron microscope .......................... 7

Figure 2.3: Inertial impactors with single and multiple stage collection system...................... 10

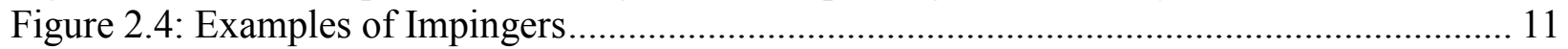

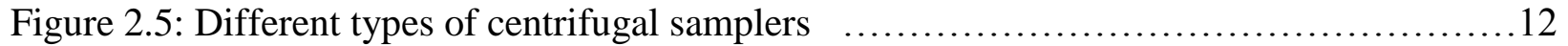

Figure 2.6: Filter sampler with different pore diameter................................................ 13

Figure 3.1: Examples of continuous domain and discrete domain ...................................... 21

Figure 3.2: Grid representation- (a) structured grid and unstructured grid, (b) Schematic of structured numerical mesh used in this project for inlet angle of $40^{\circ} \ldots \ldots \ldots \ldots \ldots \ldots \ldots \ldots \ldots . .22$

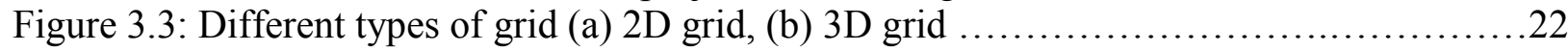

Figure 4.1: Schematic diagram of bioaerosol sampler with a microcentrifuge-tube and cyclone

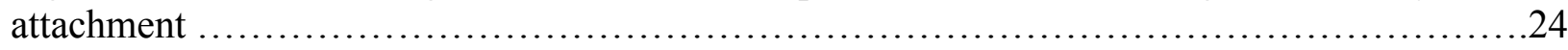

Figure 4.2: Bioaerosol sampler with (a) microcentrifuge-tube and (b) cyclone attachment

created for this project ............................................................................................ 27

Figure 4.3: The cyclone bioaerosol sampler geometry created in this project for angle (a) $30^{\circ}$, (b)

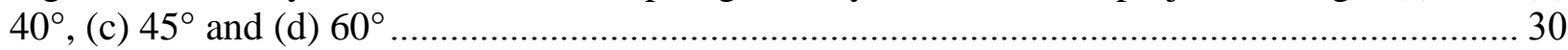

Figure 4.4: The cyclone bioaerosol sampler mesh used in this project for angle of (a) $30^{\circ}$, (b)

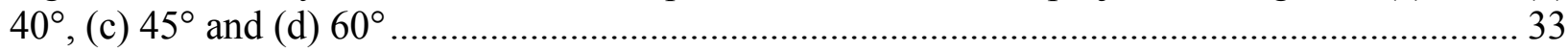

Figure 5.1: The pressure contour for four cyclone bioaerosol geometry with different angle (a)

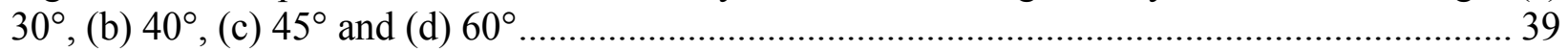

Figure 5.2: The velocity contour for four cyclone bioaerosol geometry with different angle of (a)

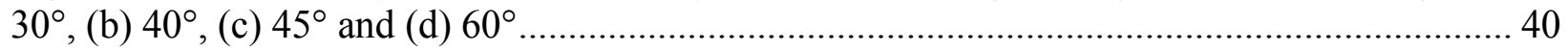

Figure 5.3: Tangential velocity contour for four cyclone bioaerosol geometry with the angle of

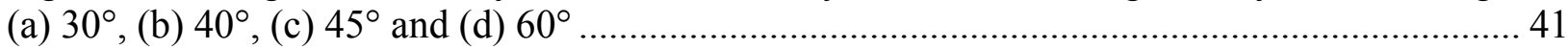

Figure 5.4: Axial velocity contour for four cyclone bioaerosol geometry with the angle of (a) $30^{\circ}$, (b) $40^{\circ}$, (c) $45^{\circ}$ and (d) $60^{\circ}$ 42

Figure 5.5: Radial velocity contours for cyclone bioaerosol geometry with an angle of (a) $30^{\circ}$,(b)

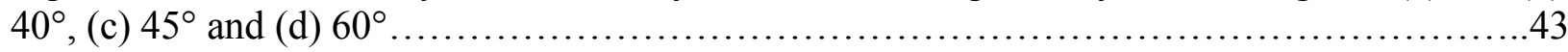

Figure 5.6: Trajectories of particle residence time for cyclone bioaerosol geometry with the angle of (a) $30^{\circ}$, (b) $40^{\circ}$, (c) $45^{\circ}$ and (d) $60^{\circ}$ 45

Figure 5.7: Trajectories of particle velocity magnitude for cyclone bioaerosol geometry with the

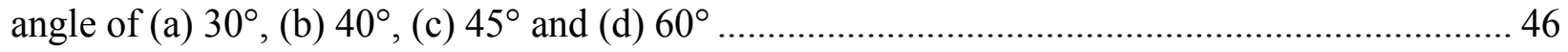
Figure 5.8: Trajectories of particle y velocity magnitude for cyclone bioaerosol geometry with

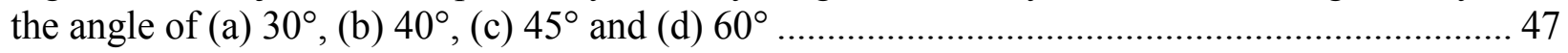

Figure 5.9: Streamline of the cyclone sampler for velocity function with the angle of $40^{\circ} \ldots \ldots . . .49$ Figure 5.10: The mean tangential velocity profiles for cyclone sampler at $\mathrm{z} / \mathrm{D}=0.5$ for different

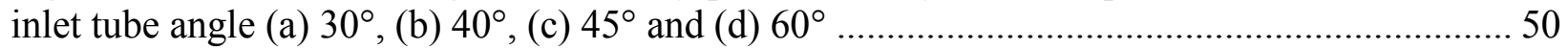
Figure 5.11: The mean tangential velocity profiles for cyclone sampler at $\mathrm{z} / \mathrm{D}=1.0$ for different

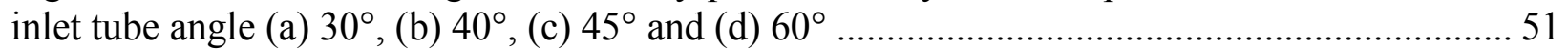
Figure 5.12: The mean tangential velocity profiles for cyclone sampler at $\mathrm{z} / \mathrm{D}=2.0$ of different

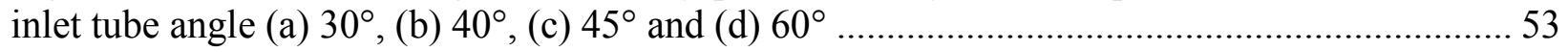
Figure 5.13: Velocity profile in vector plot for cyclone inclined of $45^{\circ}$ inlet with (a) top section, (b) bottom section and (c) slice of cyclone sampler with recirculation zone ............................ 55 Figure 5.14: Velocity vector patterns in different planes of cyclone inclined of $45^{\circ}$ inlet with (a) different plane from top to bottom section, (b) planes at conical section .57 
Figure 5.15: Collection efficiency curve with the particle diameter at flow rate of $Q=4 \mathrm{~L} / \mathrm{min}$ for cyclone sampler of different inlet tube angle (a) $30^{\circ}$, (b) $40^{\circ}$, (c) $45^{\circ}$ and (d) $60^{\circ} \ldots \ldots \ldots \ldots \ldots \ldots . . . . . . . .58$ 


\section{Chapter 1: Introduction}

This thesis is categorized into six sections. In this chapter, a brief description of the cyclone-based bioaerosol sampler will be discussed in detail. This chapter will focus on the significance of the bio-aerosol sampler and the process for accomplishing the research.

\subsection{OVERVIEW}

Cyclone-based bioaerosol samplers are extensively used in hospitals, schools, offices, industries and agronomical poultry farms to maintain indoor and outdoor air quality, as the air quality may degrade due to various microbial agents. These microbial agents may cause several health issues like allergic reactions, toxic effects, and infectious diseases [1]. Due to minimal operational costs, relatively simple geometry, and absence of moving parts and high reliability, cyclones are widely used in industries. Bioaerosol samplers are used to separate and recover the airborne or dust particles from the working environments such as schools, office, hospitals and industries. This study is focused on the microbial agent fungi. A high moisture level is favorable for fungal growth. Fungal spores are also acknowledged for asthma risk factor in indoor environments.

Airborne particles have been characterized and detected by the use of bioaerosol samplers from a long time. The main purpose of monitoring aerosol particles is to evaluate the social, economic and environmental impacts on human lives and health which occurs due to respiratory inhalation from aerosol particulates. One conventional method is used to detect and quantify fungal aerosols and airborne particles; this is the so-called short-term area grab samples. A considerable number of researchers have promoted techniques for detecting specific or "total" indoor fungal species $[1,2]$. The effect of change on the cyclone dimensions are varied with the 
change of the inlet angle or the change of the cyclone cone dimension and body diameter. Thus, researchers have developed a bioaerosol sampler that permits sample analysis without the requirement for a sample transfer step. As a big difference from industrial cyclone samplers, the bio-aerosol samplers do not require high temperature, high pressure loadings, so it is highly effective to collect airborne aerosol particles/droplets including various microbial species and airborne microorganisms. These type of bio-aerosol samplers can be directly used to supplement CFD simulation of airborne particles transport $[1,3]$. The major concern of a personal sampler is its collection efficiency at indoor conditions for different particle/droplet size.

Though the cyclone-based bioaerosol sampler has a simple geometrical design, the underlying mechanism that describes the position and velocity of particles and the interaction with fluid flow in a cyclone sampler is very intricate due to intense turbulence, dynamic anisotropy and three dimensional natures of flow [4]. The cyclone-based bioaerosol sampler performance is measured by its collection efficiency and also by the pressure drop. In our study, the pressure-drop is a less critical parameter than the industrial sampler. It becomes more clear that the inlet angle, inner wall of the collection tube and the cone geometry will play critical role for particle motions and therefore, for the collection efficiency of the sampler [1]. The effect of change on the cyclone dimensions are varied with the change of the inlet angle or the change of the other dimensions, such as inlet diameter, height of cylindrical and conical part. An inlet angle in cyclone sampler is vital for cyclone performances, as it allows the collected particles to enter the cyclone and deliver them to the discharge point of the cyclone. Though the understanding of the physics of the flow field inside the cyclone has been advanced rather quickly, the mathematical modelling of particle removal is not comprehensible yet [5]. The geometry was created by using the ANSYS WORKBENCH, and the numerical simulation was done by FLUENT 13.0.0. 


\subsection{OBJECTIVES OF THE RESEARCH}

The goal of this study is to evaluate several design options, such as inlet tube angles and different surface roughness of the collection tube inner wall through computational fluid dynamics (CFD) simulations. CFD modeling of the change of vortex core region as well as several velocity fluctuation profiles will shed light on the fundamental mechanism that influences the collection efficiency. Based on the results obtained from the simulation, the best design option will be determined.

To accomplish the project goals, the following objectives have been established:

- Generate detailed data about continuous phase flow in samplers using the state-of-the art CFD tools.

- Predict the motion of the dispersed phase particles inside a bio-aerosol cyclone sample using a 3D dispersed parcel method (DPM).

- Analyze the development and evolution of the vortex core region in the sampler.

- Assess the collection efficiency of the cyclone sampler by combining continuous phase flow and dispersed phase particles.

Four cyclone geometries have been used with different inlet tube angles in this research project. After measuring the cyclone-based bioaerosol sampler's collection efficiency, the best design option will be selected. The roughest inner wall proceeds to the best collection efficiency. The best collection efficiency leads to choose the best bioaerosol cyclone sampler design. Cyclone performances may be enhanced by the change of inlet tube angle. 


\subsection{THESIS OUTLOOK}

This thesis is divided into six chapters. Chapter 1 outlines the importance of cyclone bioaerosol samplers and the procedure for achieving the research in shortly. It also depicts the goals and objectives of this study that was designed to accomplish the result. Chapter 2 features the literature review on bioaerosol sampler, different types of bioaerosol and technologies to capture particles from working environments, which links the background to this research. Chapter 3 focuses on the theory behind the calculation and gives information for software outline. Chapter 4 gives a comprehensive description of experimental methods and boundary conditions under which the simulations were performed. It includes the geometry creation, mesh analysis and other conditions that are used to perform the simulation. A discussion of the numerical results

and theoretical calculations are discussed in Chapter 5. Finally, chapter 6 summarizes the conclusion about this work as well as recommendations for future work.

The numerical analysis was done by using the commercial software ANSYS FLUENT 13.0.0 and ANSYS CFX. These CFD tools are available on multicore processor computers at the cSETR Computational Lab, The University of Texas at El Paso. 


\section{Chapter 2: Literature Review}

Chapter 2 features the literature review of a cyclone-based bioaerosol sampler, different types, source and size of micro-organisms that may be removed from the personal sampler. It also discusses the various types of bioaerosol samplers used in sampling of bioaerosol particles, and their uses will be explained in greater detail later.

\subsection{CYCLONE BIOAEROSOL SAMPLER}

Aerosol particles are the solid or liquid particulate matters that are suspended in air or any other gaseous environments. These aerosol particles are varied with size, shape and origin. Bioaerosol particles are particles of biological origin that might release from living organisms [6]. These particles are comprised of bacteria, viruses, pollen, spores and toxin; while their sizes may vary from $0.015-0.45 \mu \mathrm{m}$ for viruses and $1.0-100 \mu \mathrm{m}$ for fungal spores. Bioaerosol sampling and characterization is complicated because of their attachment to non-viable particles and recurrent phenomena in particle accumulation. Bioaerosol sampling includes airborne particles collection and analysis of microorganisms within the sampler. These two interdependent actions are governed by aerosol physics and microbiology respectively.

Dr. Bean T. Chen, an aerosol scientist from NIOSH Health Effects Lab, has developed a new design of cyclone bioaerosol sampler while working on his research project. This cyclone bioaerosol sampler is a flow device that cleans the air from indoor and outdoor environments by taking the contaminated particles (i.e. bioaerosols and dust particles) from a working environment. Several microbial agents like bioaerosol and dust particles are responsible for various health issues which may include fatigue, allergic reactions, asthma and chronic lungs and 
several heart diseases. Figure 2.1 shows a portable bioaerosol sampler that is used in bioaerosol collection.

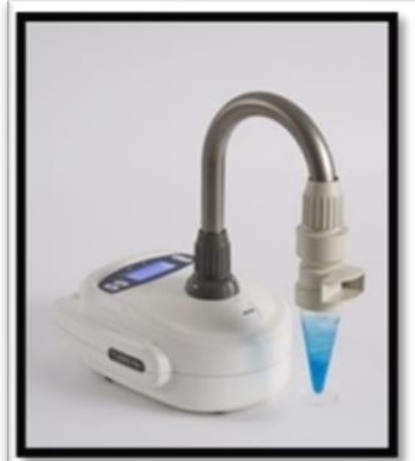

(a)

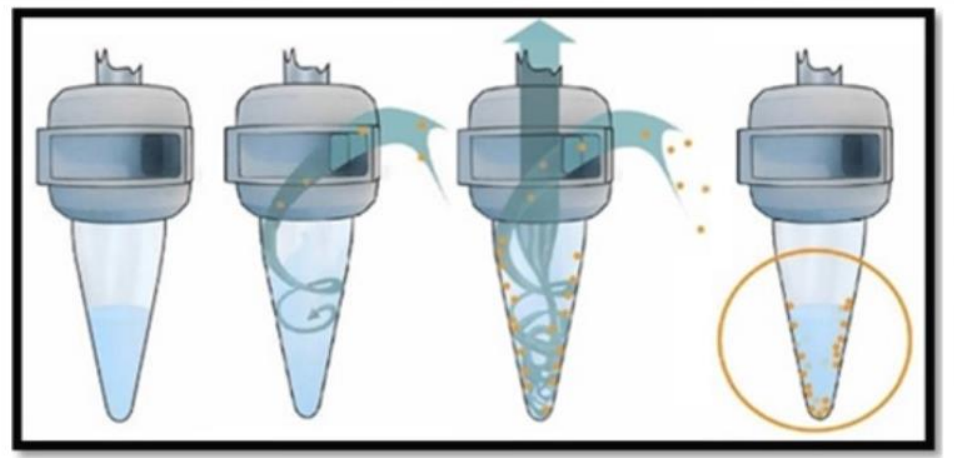

(b)

Figure 2.1: Portable bioaerosol sampler for bioaerosol collection

\subsection{BIOAEROSOLS}

Bioaerosols are the airborne particles that are suspended in air and have biological origin. These particles are small in size, and their range varies from less than one micrometer $(<1 \mu \mathrm{m})$ to one hundred micrometers $(100 \mu \mathrm{m})$. In case of outdoor air, 30 percent of all particles larger than $0.2 \mu \mathrm{m}$ are considered to be of biological origin [7]. Different types of bioaerosol particles based on particle size and concentration are described in following charts [8]:

Table 2.1: Bioaerosol sizes and concentrations in normal environment

\begin{tabular}{|c|c|c|}
\hline Type of Bioaerosols & Size $(\boldsymbol{\mu m})$ & Concentration $(\# / \mathbf{m 3})$ \\
\hline Viruses & $0.02-0.3$ & - \\
\hline Bacteria & $0.3-10$ & $0.5-1,000$ \\
\hline Fungal Spores & $0.5-30$ & $0-10,000$ \\
\hline Pollen & $10-100$ & $1-1,000$ \\
\hline
\end{tabular}


From table 2.1, it can be stated that, among the wide size range of bioaerosol, viruses are the smallest. Electron microscopy helps us to get an idea of the aerosol's morphology. Aerosol particles have diameters ranging from $1 \mathrm{~nm}$ to several hundred $\mu \mathrm{m}$. Aerosol types can be classified according to their size, which is discerned from electron microscope images as shown in figure 2.2.

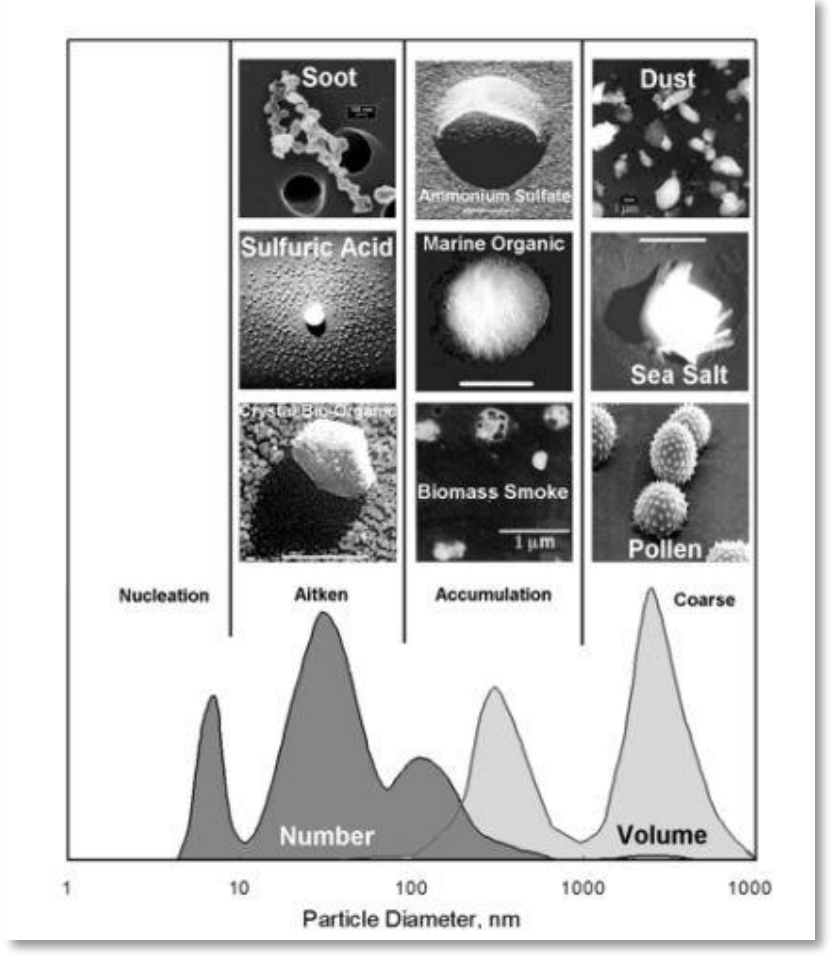

Figure 2.2: Examples of different aerosol types from an electron microscope

Since these bioaerosols have the ability to cause diseases, they are also known as pathogens. These airborne particles may be transmitted by inhalation, coughing, sneezing or either by direct contact of affected people and also by insects and wind. Several factors have impact on bioaerosol particle's activity and transmission to normal environments [9]. Some of them are the following: 
1. Relative Humidity and Temperature- Due to the hygroscopic nature of the bioaerosols, a particle's permanence is affected by their dehydration or rehydration rate. This water transfer rate is directly related to their relative humidity and temperature.

2. Oxygen- Toxic oxygen may reduce the viability of anaerobic airborne bacteria. Oxygen concentration also affects the survival rate of the bacteria by developing their growth on low oxygen conditions.

3. Radiation- Radiation effects exacerbate free-radical reactions by breaking DNA strands and protein-DNA crosslinks as well as by damaging protein, lipids, membrane and nucleic acids. Long wave radiation has less effect on bioaerosol viability.

4. Other Pollutants: The presence of ozone in outdoor environments subdued the survival rate of outdoor bioaerosols, which enhance airborne disease's transmission in indoor environments than outdoors.

\subsection{BIOAEROSOL SAMPLER SELECTION}

According to type and level of air sampling, commonly used and commercially available bioaerosol samplers can be selected [9]. These samplers are subdivided based on their mechanism of air sampling on microorganisms. A classification of the bioaerosol sampler is shown in table 2.2 based on the mechanisms and type of sampling device [7]. 
Table 2.2: Commonly used and commercially available bioaerosol samplers

\begin{tabular}{|c|c|c|}
\hline Sampler & $\begin{array}{l}\text { Manufacturer/ } \\
\text { Supplier }\end{array}$ & Notes \\
\hline $\begin{array}{l}\text { Inertial impactors } \\
\text { Air-O-Cell@ } \\
\text { Allergenco Air Sampler (MK-3) } \\
\text { Andersen Samplers, 1-, 2-, or 6-stage } \\
\text { Burkard Sampler } \\
\text { Casella Airborne Bacteria Sampler (MK-II) } \\
\text { Mattson-Garvin Slit Sampler } \\
\text { Rotorod } \\
\text { Surface Air Sampler (SAS) }\end{array}$ & $\begin{array}{l}\text { ZAAVSKC } \\
\text { ALL } \\
\text { AND } \\
\text { BUR } \\
\text { CAS } \\
\text { BAR } \\
\text { SAM } \\
\text { PBI/SPI }\end{array}$ & \multirow{4}{*}{$\begin{array}{l}\text { - Air-O-Cell and Burkard samplers - the slit } \\
\text { impactors, on microscope slides or tape } \\
\text { - SAS amplers - portable one-stage, } \\
\text { multiple-hole impactors } \\
\text { - The Reuter centrifugal sampler (RCS) - } \\
\text { portable - } d_{50}=3.8 \mu \mathrm{m} \\
\text { - The AGI-30 and the AGI-40 can only be } \\
\text { used with water-based collection fluids } \\
\text { The BioSampler can be used with } \\
\text { nonevaporative liquids (mineral oil) - it } \\
\text { permits long sampling time. This type } \\
\text { of sampler utilizes several sampling } \\
\text { principles, including centrifugal forces and } \\
\text { tangential impingement }\end{array}$} \\
\hline $\begin{array}{l}\text { Centrifugal impactors } \\
\text { BioSampler } \\
\text { Multistage Liquid Impinger } \\
\text { Reuter Centrifugal Sampler (RCS) }\end{array}$ & $\begin{array}{l}\text { SKC } \\
\text { BUR } \\
\text { BIO }\end{array}$ & \\
\hline $\begin{array}{l}\text { Impingers } \\
\text { AGI-4, AGI-30 } \\
\text { BioSampler }\end{array}$ & $\begin{array}{l}\text { AGI/HAM/MIL } \\
\text { SKC }\end{array}$ & \\
\hline $\begin{array}{l}\text { Filter samplers } \\
\text { 37-mm Cassette } \\
\text { Button Sampler }\end{array}$ & $\begin{array}{l}\text { CCO/MIL/SKC } \\
\text { SKC }\end{array}$ & \\
\hline
\end{tabular}

Table 2.2 demonstrates different types of bioaerosol samplers based on their mechanism and their common and commercial uses (i.e. Inertial impactors, Centrifugal impactors, Impingers and Filter samplers). Specific bioaerosol characteristics have great influences on accurate and suitable sampling methods. Centrifugal impactors are one of the most common bioaerosol sampler used for collecting bioaerosols. Bioaerosol samplers based on the cyclone principle are generally known as one of the centrifugal impactors [9].

\subsubsection{Inertial Impactors}

Inertial impact is one of the most common particle collection methods for bioaerosol sampling. This inertial impactor consists of a top inlet cone, a sampling impactor stage(s) which contains hundreds of precision-drilled holes and also have a base section. These holes are formed based on the cut-off size which allowed bioaerosols deposition within a certain size range, as the larger size will be placed on the first stage [10]. The base section occupies an agar media (petri 
dish) where the accumulated air particles are deposited. Since the cut-off sizes are known for the impactors, they can give acquainted size-dependent collection efficiencies at ease [11].

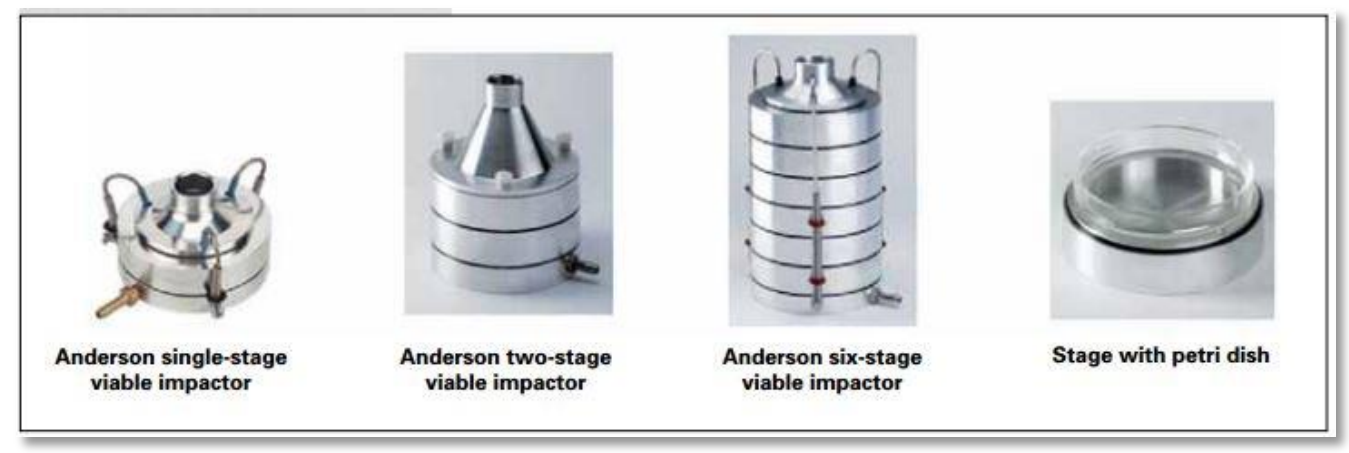

Figure 2.3: Inertial impactors with single and multiple stage collection system

Anderson impactors, a general inertial impactors used on air sampling have both singlestage and multiple stage collection systems. Based on the mechanism of inertial impactors, larger particles are deposited in the upper stages for multiple stage samplers, as the larger holes are in the upper most part of the impactors. Consecutively, smaller particles are accumulated in the lower stages [12].

\subsubsection{Impingers}

In impinger sample collection devices, the particles are collected into a liquid surface. An impinger functions like an impactor, the difference being their collection medium. An inertial mechanism is used to capture the particle, and the impinger holds the suspended particles in the fluid [9]. Though the shear force presents in the jet and turbulent liquid has detrimental effects on the bioaerosols permanence, the sample desiccation can be avoided by collecting the bioaerosol particles from liquid. Impingers are subjected to reduce the harm to bioaerosols and can maintain sampled particle durability better than impactors. 


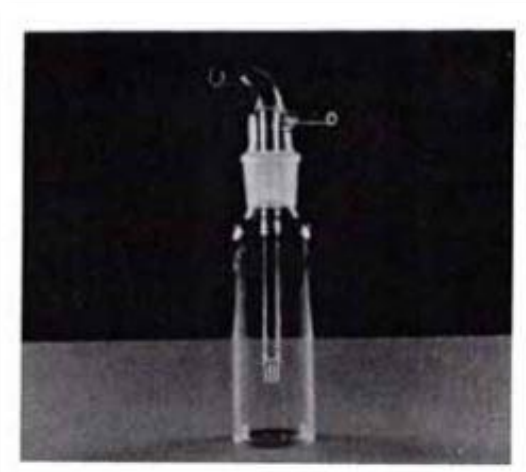

All glass AGI-30 liquid

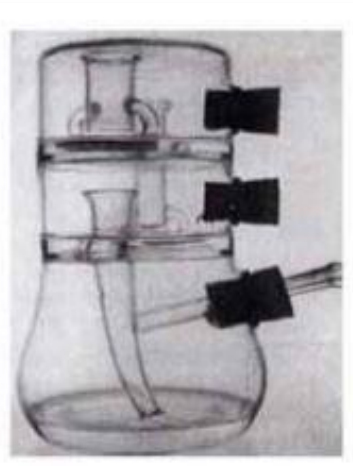

Multistage all glass liquid

Figure 2.4: Examples of Impingers

Different types of impingers are shown in Figure 2.4. AGI-30 and AGI-4 are the most conventional impingers used in bioaerosols collection. The cutoff diameter is $0.3 \mu \mathrm{m}$ for an AGI30 impinger. Because of the inertia and minor flow of the sampler, particles larger than the cutoff size are collected during sampling. Consecutively, particles smaller than $0.3 \mu \mathrm{m}$ are subjected to the major flow, decreasing the sampling efficiency [13]. The particle cut-off size mainly depends on the flow rates of the sampler [14]. In case of multistage liquid impinger (MSLI), three different sampling flow rates have been used to separate the particles into three size fractions.

\subsubsection{The Centrifugal Sampler}

The Aerojet cyclone sampler and the Biotest RCS centrifugal are the most conventional centrifugal samplers used for air sampling. Cyclones have been worked as a pre-sampler before the impactors and filters from a long time [15]. These samplers work based on the centrifugal force effects by spinning the bioaerosol particles throughout the tube axis. Larger particle are subjected to fall at the bottom of the sampler due to their inertia, while the smaller ones escape 
through the outlet. Solid particles are collected in the dust hopper residing at the bottom of the cyclone, while fluid particles cling into the wall [12].

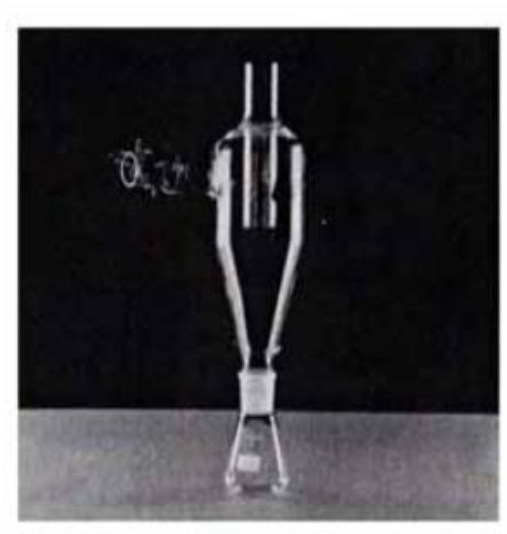

Aerojet® cyclone sampler

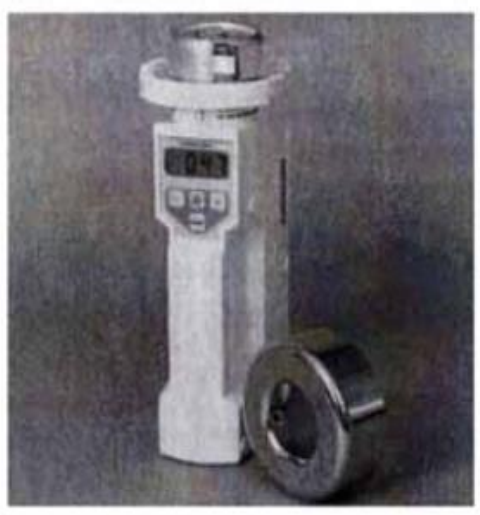

RSC Biotest $\circledast$ centrifugal sampler

Figure 2.5: Different types of centrifugal samplers

\subsubsection{Filter Sampler}

Filtration with membrane filter is another option for particle collection on bioaerosol sampling. The sampler dragged the air into the membrane filter and any bioaerosol particle smaller than the pore diameter of the filter can pass through the filter surface. An optical microscope is used to examine the collected bioaerosol samples. This method can deal with the microscopically detectable bioaerosols in extremely contaminated environments [7] [8] [9]. The main drawback of this process is the desiccation of diverse microbial species and bioaerosols. Another filtration method is using Nuclepore filters, which have large cylindrical pores (5-12 $\mu \mathrm{m})$ and have been occasionally used to give size-dependent inlets [16]. 


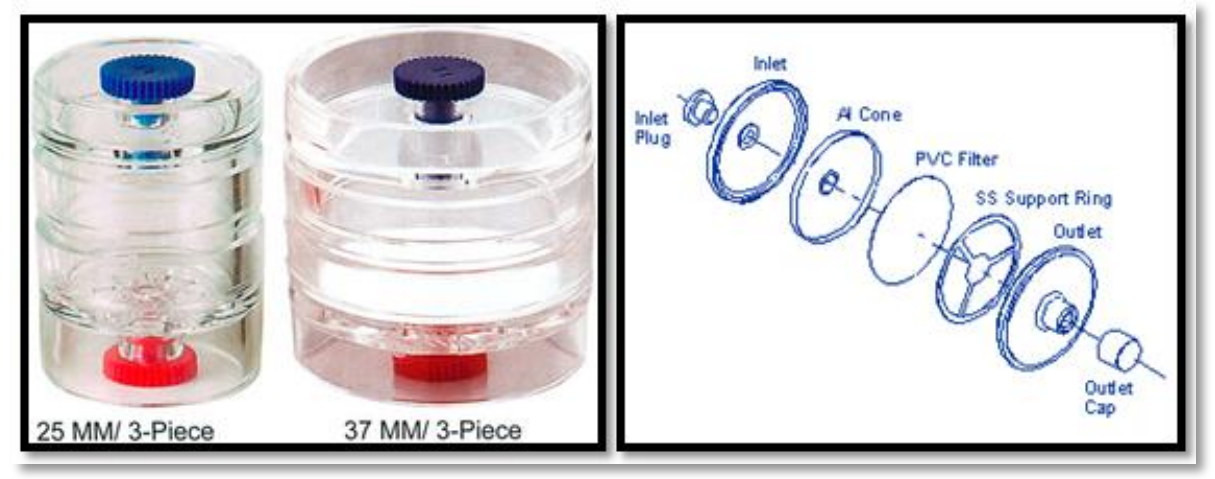

Figure 2.6: Filter sampler with different pore diameter

Selection of suitable sampler is one of the important concerns for collecting bioaerosols from environmental air. A suitable bioaerosol sampler can be chosen by viability of collected micro-organisms, total particles collected and their ability to separate bioaerosols based on size fractions [17]. Filter medium, extraction methods and microbial species results filter extraction efficiency of microbial agents [1]. Among all of the technologies for the bioaerosol air sampling, cyclone sampler gives the best result [18]. Several microbial agents may cause health issues like infectious diseases, allergic reactions, inflammation in lungs and blood vessels and may increase asthma risk factors [19]. Cyclone samplers are used to separate and recover these airborne microorganisms or dust particles from indoor and outdoor environment.

A number of researches have been conducted to characterize the flow field inside the cyclone sampler for years and new concepts are being introduced on the research of cyclone performance. Several works have been done for developing techniques on detecting specific or "total" indoor fungal species [20-23]. So, researchers have developed a personal bioaerosol sampler that does not require sample transfer step to perform sample analysis. This sampler would help researchers to accumulate breathing zone samples from working environments and biologists to utilize molecular assays for further fungal analysis [1]. Based on this analysis, 
public health specialists can apply and improve control strategies on improving air quality for indoor and outdoor environments. 


\section{Chapter 3: Numerical Method}

The numerical analysis of fluid flow gives us an extensive idea of the computational fluid dynamics (CFD), which becomes a great concern to engineers and scientists. CFD owes its popularity for predicting fluid flow, heat transfer, and chemical species transport in different environmental condition analysis [24]. With the advent of powerful parallel computers and advancement of computational techniques in recent years, CFD gives quantitative and qualitative analysis of fluid flow that is able to deal with the real life problems with better accuracy [25].

In this numerical analysis-based work, we have discussed several cases for different inlet angles with different geometry of the cyclone sampler and the particle collection efficiency of this device. To get the best collection efficiency, we have made a comparison of all obtained results from different cyclone geometry. It would take a huge cost to check all these cases in practical life. Therefore, numerical analysis provides a guideline to choose the best experimental setup to make it more cost effective [5].

\subsection{Governing EQUATIONS}

The flow field inside the cyclone sampler is a complex phenomenon in fluid dynamics problem. A combined approach on CFD and discrete element method (DEM) is exhibited on this project which explains the particle-particle and particle-fluid interactions [26-37]. For better understanding of complex particle-fluid flow, CFD-DEM approach has been extended in several studies on the gas-solid flow of a gas cyclone [38-42]. CFD analyzes the fluid dynamic phenomena by using numerical methods. Fluid Dynamics (FD) solution can be obtained by sets of governing equation. Navier-Stokes equation controlled the gas phase simulation in CFDDEM modelling [43]. The Navier-Stokes equations are nonlinear partial differential equations 
that illustrates the conservation of linear momentum for a linearly viscous (Newtonian), incompressible fluid flow [44, 45]. Density does not vary with the fluid motion in an incompressible flow.

\subsubsection{Mathematical modelling:}

Computational fluid dynamics (CFD) method is used to model the performance of cyclone bioaerosol sampler and it can anticipate the flow field characteristics and the particle trajectories inside the cyclone. The intricate swirling turbulent flow in a cyclone places great demands on the numerical techniques and the turbulence models employed in the CFD codes when modelling the collection performance.

FLUENT uses a finite volume method (FVM) to solve the governing equations. Reynoldsaveraged Navier-Stokes equations (RANS) for steady and incompressible fluid flow can be expressed as:

$$
\begin{gathered}
\rho u_{j} \frac{\partial u_{i}}{\partial x_{j}}=-\frac{\partial p}{\partial x_{i}}+\frac{\partial}{\partial x_{j}}\left[\mu\left(\frac{\partial u_{i}}{\partial x_{j}}+\frac{\partial u_{j}}{\partial x_{i}}\right)\right]+\frac{\partial \tau_{i j}}{\partial x_{j}} \\
\frac{\partial u_{i}}{\partial x_{i}}=0
\end{gathered}
$$

where, the superscripts $\mathrm{i}, \mathrm{j}=1,2$ represent the Cartesian coordinate system components, $\rho, u, p$ and $\mu$ is the fluid density, velocity, pressure and viscosity respectively and

$$
\tau_{i j}=-\rho \overline{u_{\imath} u_{j}}
$$

which is defined as the Reynolds stress tensor that indicates the turbulent fluctuations effects on the fluid flow. The dash and overbar represent the fluctuating part and a Reynolds average term. 


\section{Reynolds stress model}

The standard k- $\varepsilon$ model is inappropriate due to its certain limitations in the case of strong swirling flow [46]. Reynolds stress model (RSM) can solve the transport equations of Reynolds stress model within particular stress components and improves the isotropic turbulence of k- $\varepsilon$ model. The transport equations of Reynolds stress (RSM) model can be written as follows:

$$
\begin{gathered}
\frac{\partial \rho u_{i} u_{j}}{\partial t}+\frac{\partial}{\partial x_{k}}\left(U_{k} \partial \rho \overline{u_{i} u_{j}}\right)= \\
P_{i j}+\emptyset_{i j}+\frac{\partial}{\partial x_{k}}\left[\left(\mu+\frac{2}{3} c_{s} \rho \frac{k^{2}}{\varepsilon}\right) \frac{\partial \rho \overline{u_{i} u_{j}}}{\partial x_{k}}\right]-\frac{2}{3} \delta_{i j} \varepsilon \rho
\end{gathered}
$$

where, $\mathrm{k}$ is the turbulent kinetic energy, $\varepsilon$ is the dissipation rate of $\mathrm{k}$, pressure-strain correlation is expressed in $\emptyset_{i j}$ terms and $P_{i j}$ is the production term of $\overline{u_{i} u_{j}}$ which can be written as follows:

$$
\mathrm{P}=-\rho\left(\overline{u \cdot u}(\nabla U)^{T}+(\nabla U)\right.
$$

The turbulent dissipation rate presents within the individual stress equations. The equation for the turbulent dissipation rate $\varepsilon$ is given as:

$$
\frac{\partial \rho \varepsilon}{\partial t}+\nabla \cdot(\rho \mathrm{U} \varepsilon)=\frac{\varepsilon}{k}\left(c_{\varepsilon 1} P-c_{\varepsilon 2}\right)+\nabla \cdot\left[\frac{1}{\sigma_{\varepsilon R S}}\left(\mu+\rho C_{\mu R S} \frac{k^{2}}{\varepsilon}\right) \cdot \varepsilon\right]
$$

The model constants in these equations are

$$
c_{S}=0.22 ; c_{\varepsilon 1}=1.45 ; c_{\varepsilon 2}=1.9 ; c_{\mu R S}=0.115
$$




\section{Model Equations for Particle Motion}

The equation of dispersed particles motion can be by integrating the force balance equation in a Lagrangian reference frame. FLUENT anticipates the trajectory of a discrete phase in a Lagrangian reference frame [47]. The force balance equation of a single dispersed particle can be written (for the $\mathrm{x}$ direction in Cartesian coordinates) as

$$
\frac{d u_{p}}{d t}=F_{d}\left(\mathrm{u}-\mathrm{u}_{\mathrm{p}}\right) \frac{g_{x}\left(\rho_{p}-\rho\right)}{\rho_{p}}+\mathrm{F}_{\mathrm{x}}
$$

where, $F_{x}$ is an additional acceleration term, $u$ is the fluid phase velocity, $u_{p}$ is the particle velocity, $\rho_{\mathrm{p}}$ is the density of the particle, $g_{x}$ is the gravitational acceleration and $\mathrm{F}_{\mathrm{d}}\left(\mathrm{u}-\mathrm{u}_{\mathrm{p}}\right)$ is the drag force per unit particle mass where $\mathrm{F}_{\mathrm{d}}$ can be written as [49]:

$$
F_{D}=\frac{18 \mu}{\rho_{p} d_{p}^{2}} \frac{C_{d}}{24} R e
$$

Here, $\mu$ is the molecular viscosity of the fluid, $\rho$ is the fluid density, $d_{p}$ is the particle diameter and drag coefficient $\mathrm{C}_{\mathrm{d}}$ can be determined by Morsi and Alexander's correlation. Re is the relative Reynolds number, which is defined as,

$$
R e=\frac{\rho d_{p\left|u_{p}-u\right|}}{\mu}
$$

\section{Saffman's Lift Force}

The Saffman's lift force (lift due to shear) can also be included in coupled force term. The Saffman lift force is a generalization form from Li and Ahmadi which can be written as:

$$
\vec{F}=\frac{2 K v^{1 / 2} \rho d i j}{\rho_{p} d_{p}\left(d_{l k} d_{k l}\right)^{1 / 4}}(\vec{v}-\vec{v} p)
$$


where $\mathrm{K}=2: 594$ and $\mathrm{d}_{\mathrm{ij}}$ is the deformation tensor. This form of the lift force is intended for small particle Reynolds numbers.

\subsection{BOUNDARY CONDITIONS}

Boundary conditions (BC) are a set of conditions that specify the flow and thermal variables at the boundary of its domain. These are verily important parameter for the mathematical model and FLUENT simulations [48]. Without appropriate boundary condition settings, numerical simulation would not be able to solve the flow analysis. CFD requires proper boundary condition on the outer and inner surfaces of the fluid domain, while the inner surfaces represent fluid-structure interfaces. In general, boundary conditions are two types i.e.

i. Dirichlet boundary conditions - specify the value of the variable at the boundary, e.g. $u(x)=$ constant

ii. Neumann boundary conditions - specify the gradient normal to the boundary of the variable at the boundary, e.g. $\partial_{n} u(x)=$ constant

Usually boundary conditions depend on the type of analysis (incompressible or compressible). Another type of boundary condition is used to analyze fluid dynamics problem is called mixed type boundary conditions. These types of boundary condition specify a function of the form $a u(x)+b \partial_{n} u(x)=$ constant at the boundary.

Proper boundary conditions give an accurate solution of numerical simulation. Boundary conditions used at each face of each computational domain. It specifies physical conditions (e.g. solid wall, symmetry plane, etc.) in the boundary region and interfaces between contiguous or 
overlapped domain [49]. Inaccurate boundary condition settings can lead to a nonphysical simulation results. Therefore, defining proper and effective boundary condition is the most important part of CFD analysis.

For this research, we have used four different boundary conditions, i.e., velocity inlet at inlet, outflow at outlet, wall for boundary wall and interior BC for the interior zone.

i. Velocity inlet: Velocity inlet BC defines the velocity vectors and scalar properties of the flow at flow inlets. Since the total (or stagnation) properties of the flow are not fixed, their values may vary to give the recommended velocity distribution.

ii. Outflow: Outflow BC is used when flow velocity and pressure are not known previously. It can't be used in unsteady compressible flow and where the backflow occurs. Other flow variables are extrapolated from the interior.

iii. Wall: Wall BC is used to bound fluid and solid regions. No-slip BC is implemented on the wall in viscous flow. Based on flow field details inside the computational domain, the shear stress and heat transfer between the fluid and the wall are calculated.

iv. Interior: Interior BC is used on flow fluid through an area.

\subsection{GRID GENERATION}

Grid is generated in ANSYS WORKBENCE software for the CFD solver. Discrete domain with grid can replace the continuous domain for CFD simulation. In continuous domain, each flow function can be outlined at every point of the domain. On the other side, each flow function is defined at the grid points only in discrete domain. The discrete system is a set of coupled, algebraic equations in the discrete variables. Discrete system includes a very large number of iterative calculations which is done by the digital computer. Figure 3.1 shows the 
pressure (p) variable in 1D continuous and discrete domain. It can be said from the figure that the pressure would be defined only at the $\mathrm{N}$ grid points in terms of discrete domain where,

$p_{i}=p\left(x_{i}\right) ; \mathrm{i}=1 ; 2 ;::: ; \mathrm{N}$ and for continuous domain, it would be, $p=p(x) ; 0<x<1$

Continuous Domain

$0 \leq x \leq 1$

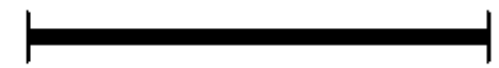

Coupled PDEs + boundary conditions in continuous variables
Discrete Domain

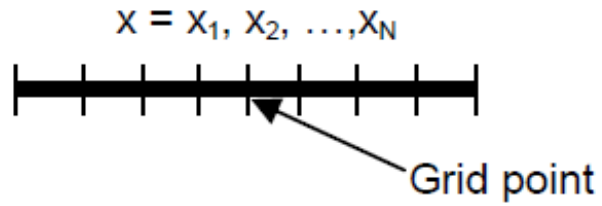

Coupled algebraic eqs. In discrete variables

Figure 3.1: Examples of continuous domain and discrete domain

Grid, a discrete geometrical representation, divides the computational domain into cells or elements on which the flow and differential problem can be solved in a most accurate and effective way. Success of the CFD analysis lies on the design and construction of a quality grid. Solution accuracy and rate of convergence is dependent on the grid size. Higher accuracy can be obtained by building finer mesh. Coordinate system gives points and directions to make a grid point. Cells are grouped into boundary zones where B.C.s is applied. Structured and unstructured, both grids are used on computational domain. Structured grid can't be used on complex geometries. A regular pattern grid arrangement is called structured grid, while irregular pattern arrangement is called unstructured grid. Unstructured grid is commonly used with the finite element method. We have used structured hexahedral grid on this thesis. 


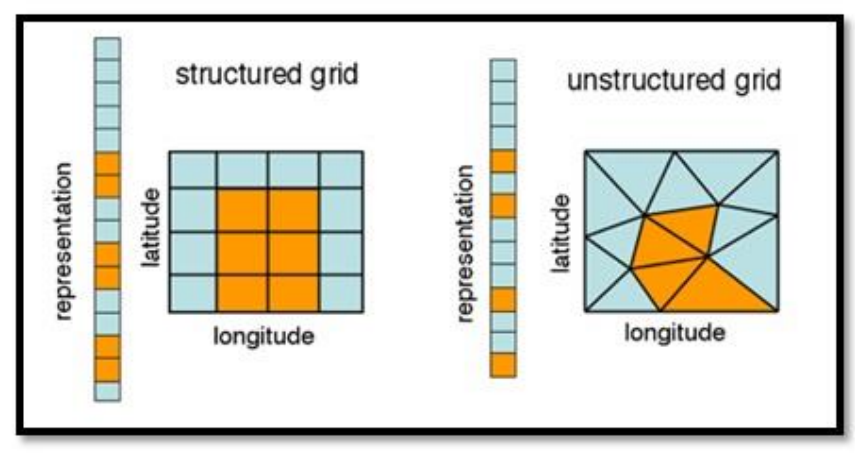

(a)

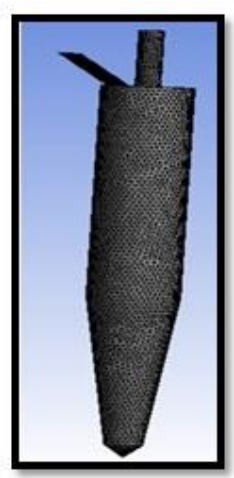

| (b)

Figure 3.2: Grid representation- (a) structured grid and unstructured grid, (b) Schematic of structured numerical mesh used in this project for inlet angle of $40^{\circ}$

Depending on the solver capability and problem settings, different types of grid are used on numerical analysis. Both 2D and 3D grid can be generated in ANSYS software. 2D grid takes less memory and less time to perform numerical analysis. A 2D grid usually considers no velocity gradients in the direction normal to the grid. 3D grid generation is the most time consuming part of the 3D simulation calculation. It takes longer computational time than $2 \mathrm{D}$ grid generation.

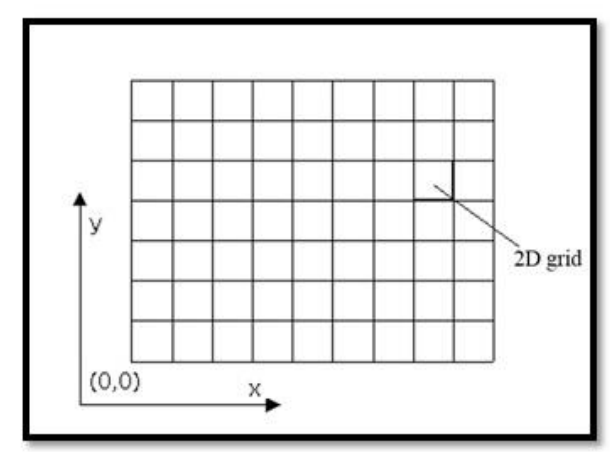

(a)

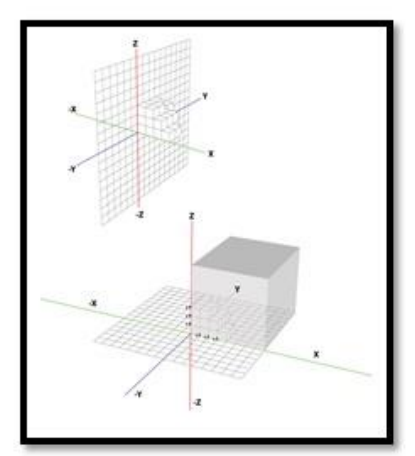

(b)

Figure 3.3: Different types of grid (a) 2D grid, (b) 3D grid 
Governing equations are not good enough to get analytical solutions of fluid flow or heat transfer problem. Finite volume method can solve the partial differential equations on fluid flow analysis. To analyze fluid flow inside a computational domain, the domain is discretized into smaller volumes or subdomains. Then the governing equations solve every discretized volume. These discretized subdomains are called elements or cells and the set of all elements are called mesh. Appropriate choice of mesh will impede the simulation error and can give us an accurate result during the flow analysis in Fluent. 


\section{Chapter 4: Methodology of Cyclone Sampler Simulation}

In this chapter the theory behind the numerical analysis of the collection efficiency of a cyclone-based bioaerosol sampler will be elaborately explained. At first, design and working principle of bioaerosol sampler will be discussed. Then four geometries with different inlet tube angle will be shown. At last the collection efficiency will be characterized for different cyclone geometry will be obtained from several equations.

\subsection{GEOMETRY OF CYCLONE-BASED BIOAEROSOL SAMPLER}

The cyclone-based bioaerosol sampler geometry was drawn by using commercial software ANSYS 13.0. The dimension of the cyclone bioaerosol sampler was chosen from a research work done by Bean T. Chen, Gregory A. Feather [1]. Figure 4.1 shows a schematic diagram of the microcentrifuge-tube and cyclone attachment.

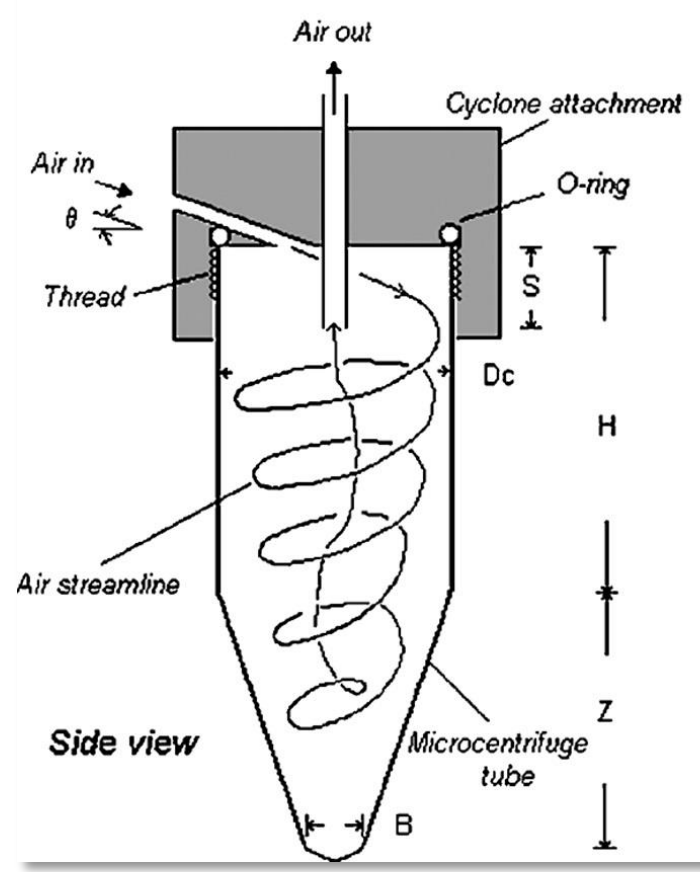

\section{Conceptual Design of a Bioaerosol Sampler}

Parameters based on the attchment

$\mathrm{Di}=$ inlet tube diameter

$\theta \quad=$ inlet tube angle

Do = outlet (vortex) tube diameter

$S=$ outlet tube length

Parameters based on the tube

Do = body diameter

$H=$ height (cylindrical patt)

$z=$ height (oo nical part)

8 a length (base part)

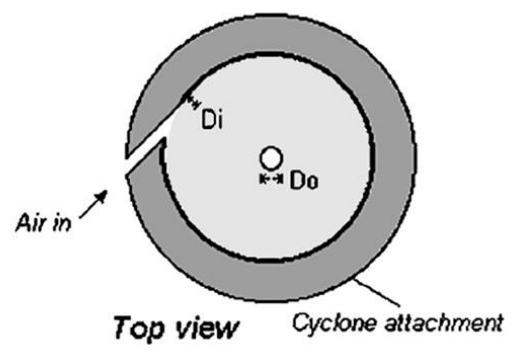

Figure 4.1: Schematic diagram of bioaerosol sampler with a microcentrifuge-tube and cyclone attachment 
According to table 4.1, all tubes had a body diameter, Dc, nearly $8.2-8.3 \mathrm{~mm}$. Heights of cylindrical portion $(\mathrm{H})$ and conical portion $(\mathrm{Z})$ could be varied depending on the capacity and type of the tube, though their total height $(\mathrm{H}+\mathrm{Z})$ kept around $44 \pm 1 \mathrm{~mm}$.

Table 4.1: Geometrical dimensions of various microcentrifuge tubes used in bioaerosol samplers

\begin{tabular}{|c|c|c|c|c|c|c|c|c|}
\hline Item & $\begin{array}{l}\text { Vendor's model } \\
\text { Number description }\end{array}$ & Attachment & $\begin{array}{l}\text { Capacity } \\
(\mathrm{mL})\end{array}$ & Type & $\begin{array}{c}\mathrm{D}_{\mathrm{c}} \\
(\mathrm{mm})\end{array}$ & $\begin{array}{c}\mathrm{H} \\
(\mathrm{mm})\end{array}$ & $\begin{array}{c}\mathrm{Z} \\
(\mathrm{mm})\end{array}$ & $\begin{array}{c}\mathrm{B} \\
(\mathrm{mm})\end{array}$ \\
\hline a & $\begin{array}{l}\text { PGC 16-8117-06 } \\
\text { Sorenson Bioscience }\end{array}$ & I & 2.0 & $\begin{array}{l}\text { Self-standing, } \\
\text { nonconical }\end{array}$ & $8.35 \pm 0.01$ & $41.13 \pm 0.05$ & $3.02 \pm 0.18$ & 0 \\
\hline b & $\begin{array}{l}\text { PGC 16-8117-26 } \\
\text { Sorenson Bioscience }\end{array}$ & & 1.7 & $\begin{array}{l}\text { Self-standing, } \\
\text { conical }\end{array}$ & $8.31 \pm 0.01$ & $26.13 \pm 0.37$ & $18.42 \pm 0.32$ & $2.97 \pm 0.11$ \\
\hline c & $\begin{array}{l}\text { PGC 16-8117-28 } \\
\text { Sorenson Bioscience }\end{array}$ & & 1.7 & Conical & $8.31 \pm 0.01$ & $26.49 \pm 0.20$ & $18.42 \pm 0.21$ & $3.02 \pm 0.09$ \\
\hline d & $\begin{array}{l}\text { PGC 16-8114-04 } \\
\text { Porex TC-514 }\end{array}$ & II & 1.5 & Conical & $8.32 \pm 0.01$ & $24.28 \pm 0.17$ & $19.29 \pm 0.20$ & $3.03 \pm 0.09$ \\
\hline e & $\begin{array}{l}\text { PGC 16-8115-24 } \\
\text { Porex } 515\end{array}$ & & 1.5 & Conical & $8.27 \pm 0.03$ & $24.48 \pm 0.19$ & $19.09 \pm 0.32$ & $2.99 \pm 0.10$ \\
\hline $\mathrm{f}$ & $\begin{array}{l}\text { COR } 430915 \\
\text { Corning }\end{array}$ & III & 2.0 & $\begin{array}{l}\text { Self-standing, } \\
\text { nonconical }\end{array}$ & $8.21 \pm 0.03$ & $39.72 \pm 0.13$ & $3.79 \pm 0.15$ & 0 \\
\hline g & $\begin{array}{l}\text { COR } 430909 \\
\text { Corning }\end{array}$ & & 1.5 & Conical & $8.16 \pm 0.01$ & $25.62 \pm 0.17$ & $18.24 \pm 0.17$ & $2.94 \pm 0.08$ \\
\hline h & $\begin{array}{l}\text { SIM T334-5 } \\
\text { Simport }\end{array}$ & & 1.5 & Conical & $8.19 \pm 0.03$ & $25.76 \pm 0.17$ & $18.30 \pm 0.20$ & $2.96 \pm 0.12$ \\
\hline $\mathrm{i}$ & $\begin{array}{l}\text { SIM T334-7 } \\
\text { Simport }\end{array}$ & & 2.0 & $\begin{array}{l}\text { Self-standing, } \\
\text { nonconical }\end{array}$ & $8.22 \pm 0.05$ & $39.39 \pm 0.22$ & $4.35 \pm 0.23$ & 0 \\
\hline
\end{tabular}

Since all the tube dimensions are definite for the cyclone designs, the inlet and outlet of the attachment were designed based on the conventional cyclone. The body diameter of the bioaerosol sampler used in this research is $\mathrm{D}$ or $\mathrm{D}_{\mathrm{c}}=8.31 \mathrm{~mm}$, height of cylindrical part, $\mathrm{H}=$ $26.13 \mathrm{~mm}$ and height of conical part, $\mathrm{Z}=18.42$. All the tube dimensions are almost similar for the bioaerosol sampler used in table 4.1 other than the thread. The thread was designed based on the type of tube. Though the inlet diameter is fixed, we have used different inlet tube angle on this thesis. This inlet tube angle could be varied from $30^{\circ}$ to $60^{\circ}$ [5]. 
From the sharp-cut cyclone, the cut-off diameter equation is,

$$
D_{50}=e^{a} D_{c}^{b} Q^{1-b}
$$

where, $D_{50}$ is the cyclone cut-off diameter, $\mathrm{a}=1.447$ and $\mathrm{b}=2.131$ used in sharp-cut cyclone $[51]$.

\subsection{CYCLONE-BASED BIOAEROSOL SAMPLER DESIGN}

The cyclone bio-aerosol sampler comprised with a microcentrifuge tube and a cyclone attachment. These tubes are mainly used for sample sonicating, homogenizing, separating and sample transferring in molecular analysis. This tube is cylindrical in shape and tapering to the conical shape at the bottom. A cyclone attachment attached on the top of the microcentrifuge tube, has an inlet and outlet. The attachment is designed in this way so that it can easily fit the tube and can accomplish a desired $50 \%$ cutoff size $\left(\mathrm{D}_{50}\right)$.

To enhance the particle collection in the tube and initialize the particle impaction onto the wall, instead of placing tangentially, the cyclone inlet makes an inclined angle (of $30^{\circ}, 40^{\circ}, 45^{\circ}$ and $60^{\circ}$ ) with the horizontal axis. Figure 4.2 shows the bioaerosol sampler with microcentrifugetube and a cyclone attachment which has been created for this project. 


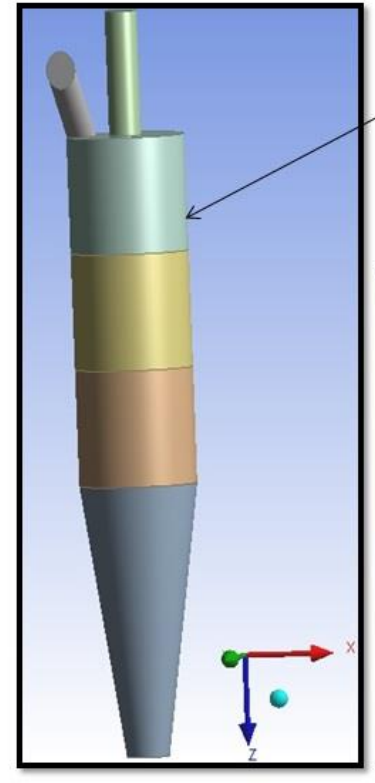

(a)
Micro-centrifuge tube

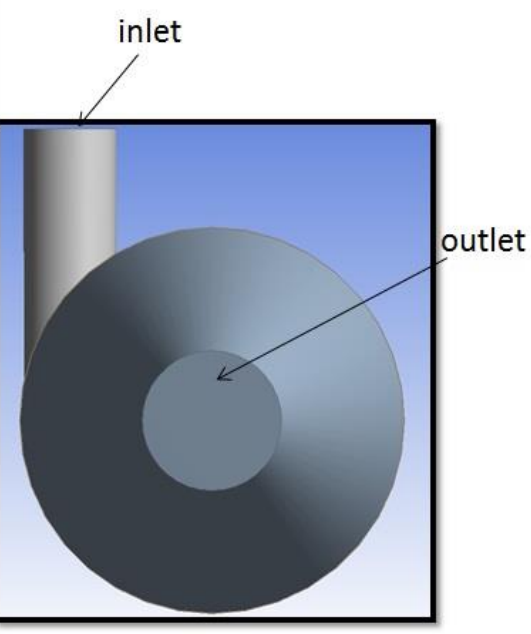

(b)

Figure 4.2: Bioaerosol sampler with (a) microcentrifuge-tube and (b) cyclone attachment created for this project

\subsection{WORKING PRINCIPLE OF CYCLONE BIOAEROSOL SAMPLER}

The self-contained, operation flexible and highly reliable cyclone bio-aerosol sampler is used to separate and recover the airborne or dust particles from the working environment, thus minimize the health risk of workers. Bioaerosol sampler works based on cyclone principle is shown in figure 4.1. The working principle of cyclone bioaerosol sampler as given as follow:

Air tangentially enters from the topmost part of the micro centrifuge tube and removed from the tube axis by creating a double vortex flow within the tube.

Due to this strong vortex flow, denser particle in the flow are experienced to centrifugal forces. 
Particles which have sufficient inertia have an impact on the tube walls.

$>$ Some of the particles are retained on the walls and due to the secondary flow in the boundary layers; other particles migrate to the bottom of the tube.

$>$ The vortex flow headway to the bottom of the cyclone sampler that reduces the rotational radius of the stream thus separates smaller particles from main flow.

$>$ Two types of vortices formed inside the cyclone- inner vortex and outer vortex. Outer vortex spirals downward from the inlet along the cyclone wall towards the bottom end and inner vortex starts from the cone apex then spirals upward to the outlet.

The purpose of this cyclone-based bioaerosol sampler design is to collect more particles in the microcentrifuge tube, which could be used as the collection receptacle for further analysis.

Cyclone attachment placed on the top of the microcentrifuge tube is used to get a desired $50 \%$ cutoff size $\left(\mathrm{D}_{50}\right)$.

Cyclone inlet makes an inclined angle with the horizontal axis to enhance the particle collection in the tube within specific size range and initialize the particle impaction onto the wall.

\subsection{PROBLEM DESCRIPTION OF CYCLONE-BASED BIOAEROSOL SAMPLER}

The present study deals with the cyclone-based bioaerosol sampler as shown in figure 4.1. The geometrical dimensions of four cyclone sampler used in this study are given in table 4.2. All of their dimensions are the same only the inlet tube angle is different for the four cyclone sampler. From figure 4.1, it is seen that the inlet angle of air sucking into the sample is denoted by $\theta$, different angle will directly impact the tangential and axial velocity components. The inlet section is changed by using $30^{\circ}, 40^{\circ}, 45^{\circ}$ and $60^{\circ}$ with respect to the microcentrifuge 
tube. All the cases will be simulated by using different inlet tube angle on the flow field of cyclone sampler and overall collection efficiencies will be measured.

Table 4.2: Geometric configuration of Cyclone studied for this project

\begin{tabular}{|l|l|}
\hline Dimension & Size(mm) \\
\hline Body diameter, D or $\mathrm{D}_{\mathrm{c}}$ & 8.31 \\
\hline Gas outlet diameter, $\mathrm{D}_{\mathrm{o}}$ & 2.24 \\
\hline Gas inlet diameter, $\mathrm{D}_{\mathrm{i}}$ & 1.99 \\
\hline Outlet tube length, $\mathrm{S}$ & 2.91 \\
\hline Height of cylindrical part, $\mathrm{H}$ & 26.13 \\
\hline Height of conical part, $\mathrm{Z}$ & 18.42 \\
\hline Length of Base, $\mathrm{B}$ & 2.97 \\
\hline
\end{tabular}

Table 4.3 shows the different inlet tube angle used in this study. We have created four cyclone samplers with different inlet tube angle to observe the difference of flow pattern and particle collection efficiency.

Table 4.3: Geometrical inlet tube angle of cyclone studied

\begin{tabular}{|l|l|}
\hline Dimension & Angle $\left(^{\circ}\right)$ \\
\hline Inlet tube angle, $\theta$ & $40^{\circ}$ \\
& $30^{\circ}$ \\
& $45^{\circ}$ \\
& $60^{\circ}$ \\
\hline
\end{tabular}

In this research, we have used a 3D full order high-fidelity CFD tool angle to simulate the particle flow inside a bio-aerosol cyclone sampler to evaluate different design options for optimization of the collection efficiency of particles. 


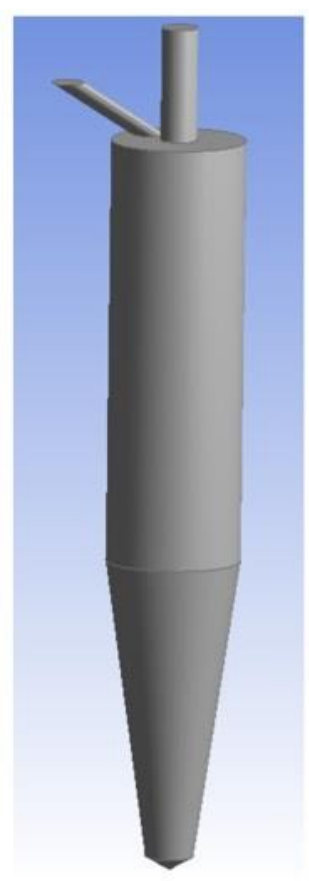

(a)

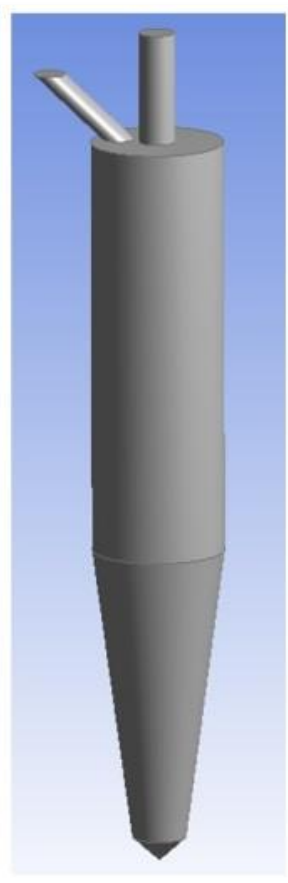

(b)

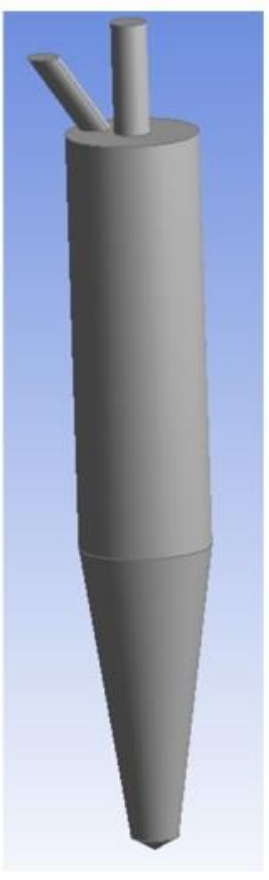

(c)

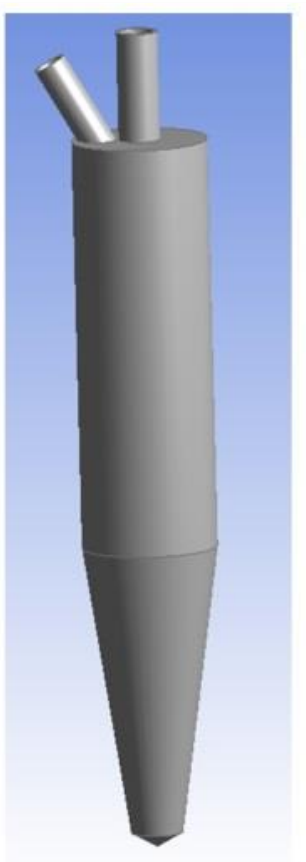

(d)

Figure 4.3: The cyclone bioaerosol sampler geometry created in this project for angle (a) $30^{\circ}$, (b) $40^{\circ}$, (c) $45^{\circ}$ and (d) $60^{\circ}$

Cyclone bioaerosol sampler geometries with different inlet tube are shown in figure 4.3. These geometries have been created in ANSYS WORKBENCH and meshing has been done using ANSYS MESHING.

Personal aerosol samplers are widely used to measure the occupational exposure of airborne materials i.e. fungi and bacteria. These can also be used for indoor air quality analysis in schools, offices, homes and other locations. Samples collected in the bioaerosols, are usually recognized by culturing or examining samples with a microscope. Molecular and immunological techniques are becoming an important application on bioaerosols analysis [2]. We have chosen different geometry based on cyclone inlet angle of $30^{\circ}, 40^{\circ}, 45^{\circ}$ and $60^{\circ}$ to observe the particle collection efficiency in the tube. Then we will compare all the results to find the best case scenario. 


\subsection{CALCULATION OF COLLECTION EFFICIENCY FOR CYCLONE SAMPLER}

Cyclone samplers are largely depending upon the inertial effects for aerosol collection. Cyclone collection efficiency can be described by two parameters i.e. aerodynamic diameter and geometric standard deviation (GSD) [7]. Aerodynamic diameter is the diameter of the spherical particle at which the collection efficiency is 50\% (called the 50\% cut-off diameter, or $d_{50}$ ). The other parameter is the GSD of the collection efficiency curve, which is also known as sharpness. When sharpness is between 1.3 and 1.5, it gives a well-defined particle size cut-off but greater than 2 sharpness value could not accomplish a definitive particle separation.

An ad hoc value of inlet angle the roughness does not guarantee the optimal collection efficiency. The collection efficiency was calculated based on the following equations, which is known as Lapple model equations and recommended in the literature [50-52].

$$
\left(d_{p 50 \%}\right)=\sqrt{\frac{9 \mu b}{2 \pi N_{e} V_{i} \rho_{p}}}
$$

Where, $\mathrm{b}=0.25 \mathrm{D}_{\mathrm{i}} ; N_{e}=\frac{H+0.5 Z}{D_{i}} ; V_{i}=$ velocity at inlet $=20 \mathrm{~m} / \mathrm{s} ; \mathrm{D}_{\mathrm{i}}=$ inlet diameter.

Collection efficiency $n$ can be written as follows:

$$
n=\frac{1}{1+\left(\frac{\left(d_{p}\right)_{50}}{d_{p}}\right)^{2}}
$$




\subsection{CYCLONE SAPMLER MODELING IN WORKBENCH}

A 3D geometry of the cyclone-based bioaerosol sampler has been drawn by the design software ANSYS Workbench 13.0. We had to create four different bioaerosol samplers with different inclined inlet tube. The cyclone sampler has a microcentrifuge tube and an attachment placed on the top of the tube. A vortex finder region is situated beneath the outlet of the sampler. Distributors are placed near the inlet and outlet. Total height of the sampler is 44.55 $\mathrm{mm}$ and the length of the vortex finder is $2.91 \mathrm{~mm}$.

For design of cyclone sampler, the diameter of the tube, inlet diameter and the length of the outlet are assumed to be constant. Dimension of the cyclone sampler are varied by the position of inlet tube angle, tube diameter and the total height of the sampler. The mesh operation was done at the end to make the geometry prepared for numerical fluid flow analysis within the area. The sampler domain is discretized into finite volumes using hex dominant mesh. Then meshing the face with quadrilateral elements was done. Finally the numerical grids were generated using the mesh building code ANSYS WORKBENCH MESHING and have around 284023 hexahedral cells for geometry with inlet angle of $40^{\circ}$. Then the geometry has a finer mesh with no skewed elements to solve the fluid flow inside a computational domain. Four different bioaerosol samplers according to their mesh sizes for are shown on table 4.4.

Table 4.4: Mesh sizes for four bioaerosol samplers used in this study

\begin{tabular}{|c|c|}
\hline Cyclone Geometry when inlet angle is & Elements \\
\hline$\theta=30^{\circ}$ & 447737 \\
\hline$\theta=40^{\circ}$ & 284023 \\
\hline$\theta=45^{\circ}$ & 453961 \\
\hline$\theta=60^{\circ}$ & 468661 \\
\hline
\end{tabular}




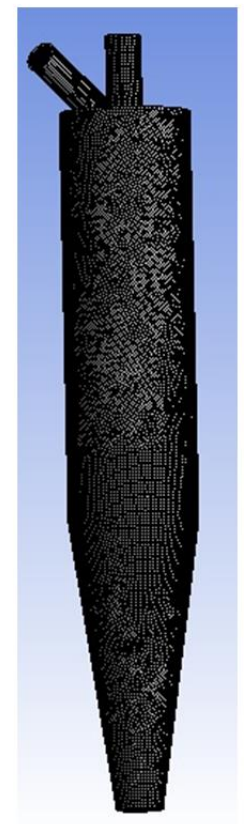

(a)

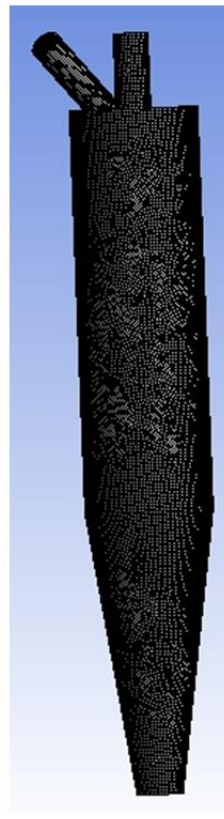

(b)

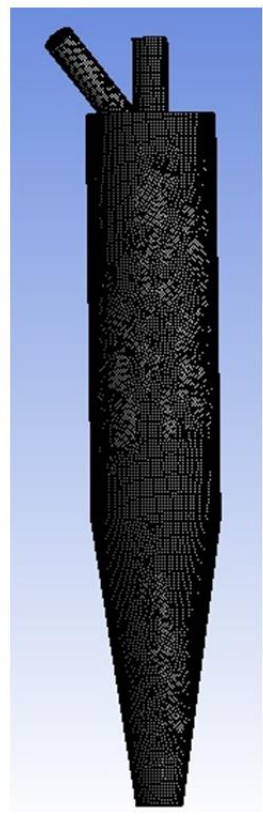

(c)

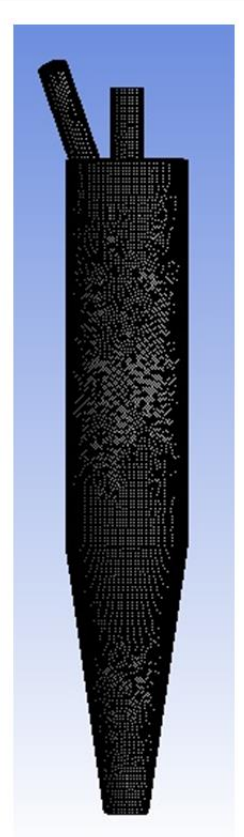

(d)

Figure 4.4: The cyclone bioaerosol sampler mesh used in this project for angle of (a) $30^{\circ}$, (b) $40^{\circ}$, (c) $45^{\circ}$ and (d) $60^{\circ}$

Computational grids used in this project have 447737 and 284023 cells for inlet tube angle of $30^{\circ}$ and $40^{\circ}$ respectively. The other mesh used in this study for four bioaerosol samplers are shown through table 4.4 and later on figure 4.4 . 


\subsection{CFD SIMULATION OF CYCLONE SAPMLER}

A commercially available CFD code, FLUENT is used to solve the discretized equations (conservation of mass, momentum and energy equations) by finite volume formulation. Once the bioaerosol sampler modeling had done, boundary condition was implemented. In this study, a velocity inlet boundary condition was applied at inlet; outflow BC at outlet and no slip BC at the walls. Air was considered as continuous phase material and waterliquid as a dispersed phase material in this study. The Eulerian-Lagrangian approach has been employed to simulate the cyclone sampler. Numerical simulations were done by the Eulerian reference frame for continuous gas phase flow and by Lagrangian method for dispersed phase particle trajectories. 3-D, unsteady and incompressible fluid flow in Eulerian formulation was introduced in this investigation. The Lagrangian approach resolves the transport equations of continuous phase in Eulerian frame and then integrates the dispersed phase equations by tracking individual spherical particles through the converged flow field. The stochastic Particle Tracking Model outlined the particle motions by tracking particle trajectories in Lagrangian frame of reference. By keeping track on the number of particles escaping through the outflow, the collection efficiency could be measured.

There are a lot of turbulence model available in FLUENT solver. As k- $\varepsilon$ model has some limitations to swirling turbulent flow, Reynolds stress turbulence model (RSTM) was used to simulate the cyclone-based bioaerosol sampler. The standard wall function is chosen for the near-wall treatment. Because of the small size (i.e. $0.5-16 \mu \mathrm{m})$ of the injected particle in the sampler, the velocity fluctuation of the small particles on turbulent dispersion cannot be overlooked. The fluctuating gas velocities can be determined by using a discrete random walk (DRW) model which is based on the stochastic tracking scheme. 
The SIMPLEC (Semi-Implicit Method for Pressure-Linked Equations-Consistent) scheme for pressure - velocity coupling has been used in finite volume method to discretize the partial differential equations and second order upwind scheme is used in the solution method for the control volume variables. The inlet velocity is considered as $20 \mathrm{~m} / \mathrm{s}$ for this study [4]. The maximum number of integration steps were $5 \times 10^{5}$ and a length scale of $0.005 \mathrm{~m}$ were chosen for the dispersed particle trajectories. Particle time step size of $0.001 \mathrm{~s}$ with an automated tracking scheme was selected for the numerical experiments and an accuracy of $10^{-5}$ was performed for accuracy control. In inlet, the turbulent intensity was set as 0.1 and Reynolds stresses are specified as normal and shear stresses which is $666.67 \mathrm{~m}^{2} / \mathrm{s}^{2}$ and 0 respectively.

\subsection{Numerical Modeling}

A highly reliable CFD modeling can help us to understand the physics inside the flow field of cyclone sampler. Appropriate computational modeling is essential to comprehend the flow physics of the system and determine the underlying methodology of CFD. It can accurately predict the behavior of flow with the help of powerful digital computers. The numerical solution of computational flow modeling includes resolving the fluid dynamics phenomena in details. Grid refinement is important to determine the ordered discretization error in CFD simulation. Computational problem size can be augmented by grid refinement which will increase the need for parallel computing. The multiphase flow considered here is isothermal system which does not involve temperature change, even though temperature variation could play a role to change the deposition of particle inside the tube. The flow is 3D incompressible gas-droplet/particle twophase interactions. 
Problem with mathematical modelling of flow field includes for indoor air measurement, the inlet air velocity is large enough that the flow is turbulent in the sampler. Turbulence models are based on statistical analysis as it is becoming an interesting alternative on CFD modelling. In past years, different turbulent models, e.g. standard $k-\epsilon$ model, $k-\omega$ model, and RNG $k-\epsilon$ have been applied to RANS to model cyclone flow inside the sampler [53]. Large disparate results compared to experimental data have been reported in published literature (e.g. Chen 1997). For this reason, different turbulent models have been justified for cyclone flow as a first step of the design evaluation. On the other hand, the numerical simulation efficiency mainly depends on the form of the turbulence modelling. As standard $k-\varepsilon$ turbulence model conducts to impracticable tangential velocities and enormous turbulent viscosities, previous studies indicate the inability of standard $\mathrm{k}-\varepsilon$ turbulence model to simulate the highly swirling turbulence flow [54]. Since Reynolds stress turbulence model (RSTM) justifies the stream curvature effects, rotation and the swirling flow effects, this (RSTM) model is appropriate for highly anisotropic flows. So, the preciseness of the numerical simulation can be improved by Reynolds stress turbulence model (RSTM) [55-57].

We carried out CFD analysis for various design option to evaluate the particles collection efficiency using Fluent 13.0 software. Several velocity fluctuation profiles will describe fundamental mechanism inside the bio-aerosol cyclone sampler. Finally, the best design option will be chosen for cyclone sampler based on their particle collection efficiency.

\subsubsection{Solver Settings}

Numerical analysis was performed by using a commercially available CFD code, FLUENT 13.0 that utilizes the finite volume formulation to perform segregated or coupled calculations with the help of conservation of mass, momentum and energy equations [50]. The 
flow is assumed to be turbulent. We have chosen RSM in this study. Velocity distribution is calculated by using the pressure based solver for steady-state flow. Energy equation was enabled for this numerical analysis from the solver panel. The temperature assumed to be constant along the velocity distribution through the cyclone sampler. Fluid properties were maintained mixinglaw for mixture-species which means that the properties such as, density, specific heat capacity, thermal conductivity, and viscosity were composition dependent. Air and water-liquid were considered as continuous and dispersed phase material in this study. After calculation of continuous phase flow on Eulerian reference frame, the particle trajectories for dispersed phase were computed through Lagrangian method. Spherical particles are used in discrete phase modelling. 


\section{Chapter 5: Results \& Discussion}

The numerical results on the performance of cyclone-based bioaerosol sampler for different inlet tube angle are presented in this chapter. To validate the continuous phase model, we have used RSM for better accuracy over other models and the flow will be specified with different velocity fluctuation profiles. Then the particle trajectories calculations for the dispersed phase will be undertaken until the particles reach at the bottom of the sampler. We have used Eulerian-Lagrangian approach to simulate the cyclone sampler. Four different cyclone geometries have been studied. At last, a comparison will be made for all these four cyclone geometries to find the best design option.

\subsection{RESULTS OF CONTINUOUS PHASE SIMULATION ON CYCLONE SAMPLER}

CFD is an important tool to describe the details of flow field of a cyclone sampler. The RSM used in this study described the turbulence model and DPM described the dispersed phase. RSM model is very effective to comprehend the flow field of cyclone sampler. Continuous phase model validates the flow individualizations with different velocity fluctuation profiles. RSM is used to simulate the fluid flow field inside the cyclone sampler and the fluid flow behavior is reviewed in the terms of velocity components of the sampler.

\subsubsection{Pressure field}

The pressure contour plot shows that the static pressure decrease from wall to center and due to high swirling velocity in the central region, a negative pressure region found in the vortex finder. So, if any particle enters into this zone is supposes to escape through vortex finder 
region. Pressure near the walls is the largest one and with the increasing radius of the sampler, pressure decreases and reaches to a minimum value in the central axis. Pressure gradient in radial direction is greater than the axial and tangential directions. The contours plots of pressure using RSM with four cyclone geometries are presented in figure 5.1.

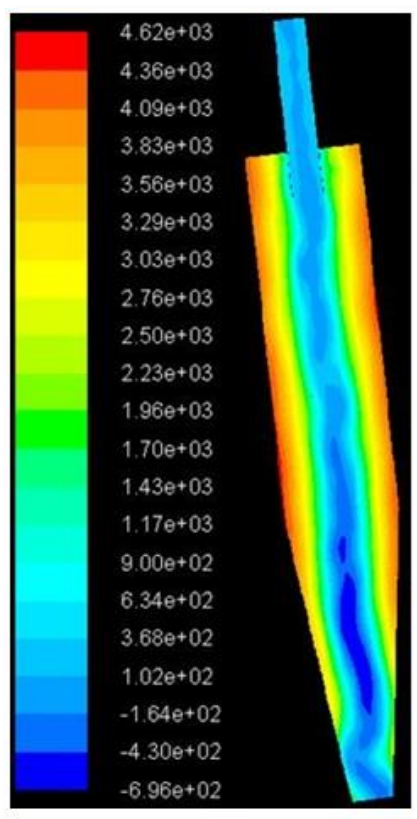

(a)

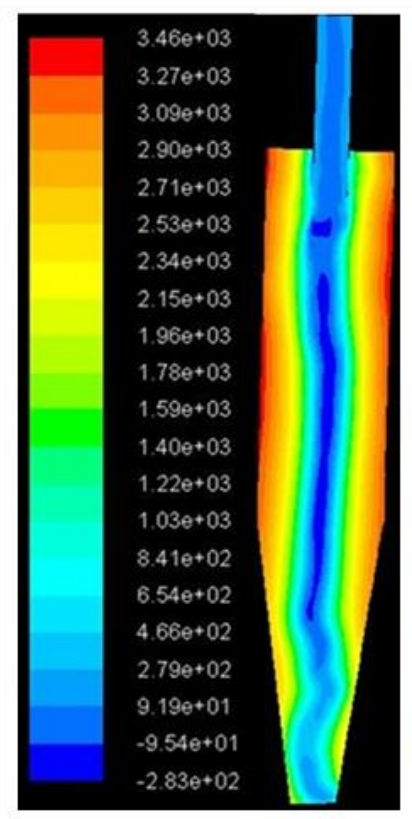

(b)

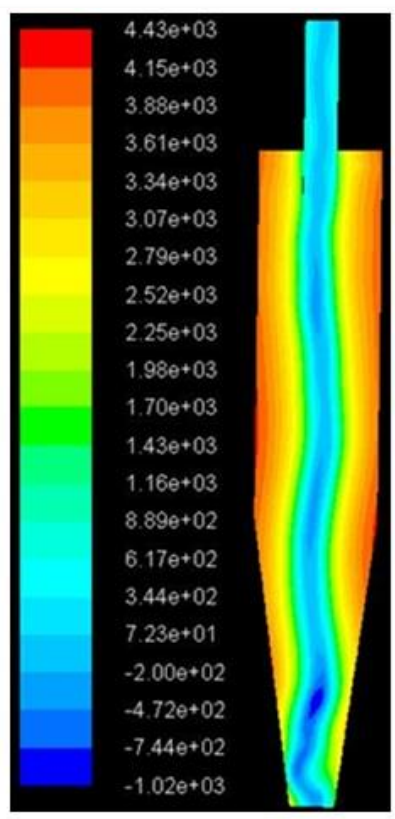

(c)

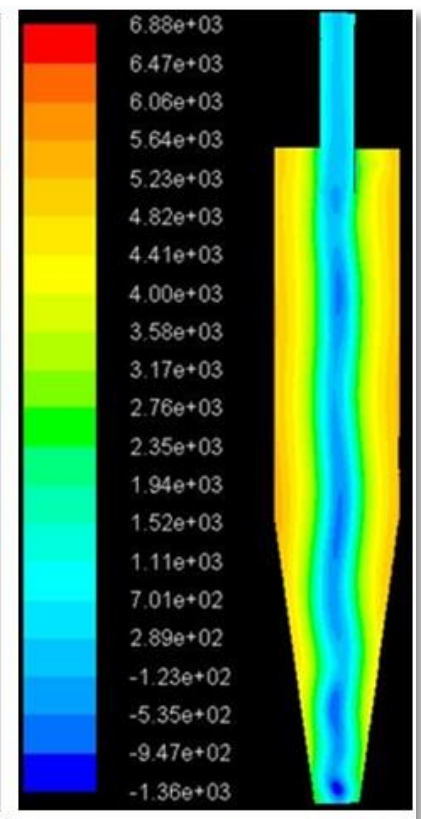

(d)

Figure 5.1: The pressure contour for four cyclone bioaerosol geometry with different angle (a) $30^{\circ}$, (b) $40^{\circ}$, (c) $45^{\circ}$ and (d) $60^{\circ}$

\subsubsection{Velocity field}

We have used different inlet angle to observe the velocity fluctuation occurs inside the flow field of cyclone. RSM used in this study to verify the cyclone sampler performances with different inlet tube angles. Figure 5.2 shows the velocity distribution for the cyclone sampler with the $30^{\circ}, 40^{\circ}, 45^{\circ}$ and $60^{\circ}$ inlet tube angles. Velocity contours are one of the important variables in fluid dynamics phenomena. Swirling flow development in fluid dynamics is attained 
by the numerical simulations in fluid dynamics. Their effects on the velocity distribution on figure 5.2 for different inlet section angles are observed. There is recirculation occurred inside the cyclone due to their high swirling velocity. Smaller velocity is found near the vortex finder region. As the flow moves towards the conical section of the cyclone, the flow became constricted due to the curtailed flow area. Therefore, the velocity increases as the particle separation happens at the main separation area.

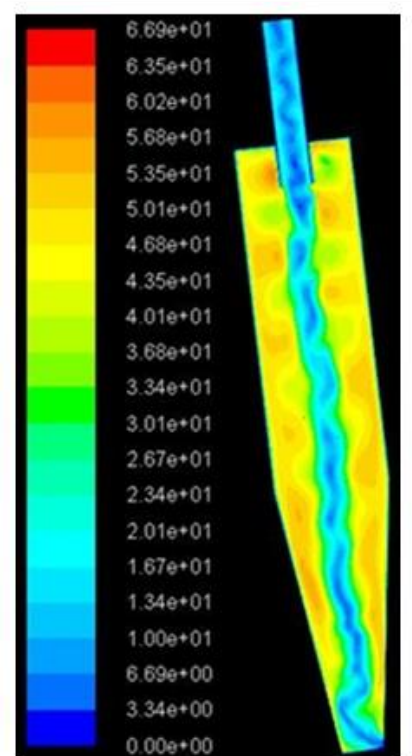

(a)

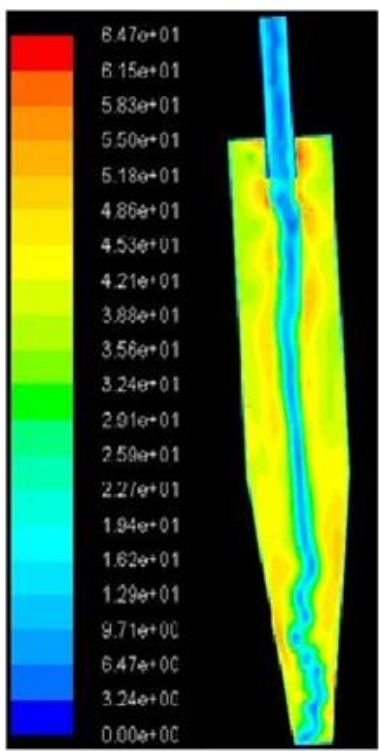

(b)

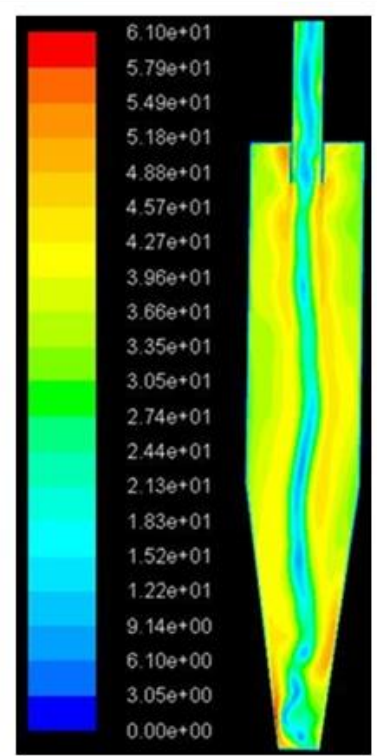

(c)

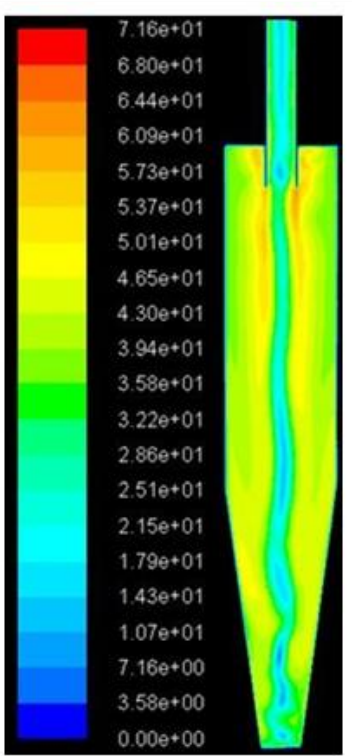

(d)

Figure 5.2: The velocity contour for four cyclone bioaerosol geometry with different angle of (a)

$$
30^{\circ}, \text { (b) } 40^{\circ} \text {, (c) } 45^{\circ} \text { and (d) } 60^{\circ}
$$

\subsubsection{Tangential velocity}

Tangential velocity plays an important role in three-dimensional incompressible fluid flow of cyclone sampler because of their approach of generating centrifugal force. As the tangential velocity is the prevalent component in the sampler, so the contours plot of the velocity 
magnitude and tangential velocity components are almost identical. The lowest value of these velocity components are found near the wall and in the vortex finder region. When the gas enters through the inlet is experienced the centrifugal force. Due to this, they accelerate up to 2-3 times of the inlet velocity value and when the gas spins down; it decreases the velocity magnitude gradually. The tangential velocity distribution distinguishes into two regions, namely, a forced vortex at the center of the cyclone and a free vortex at the surrounding region of forced vortex. Due to larger centrifugal forces in inner region, tangential velocity gets higher which results in higher particle collection efficiency. We are getting higher tangential velocity for inlet angle of $30^{\circ}$. The contours plots of tangential velocity of the cyclone are presented in figure 5.3.

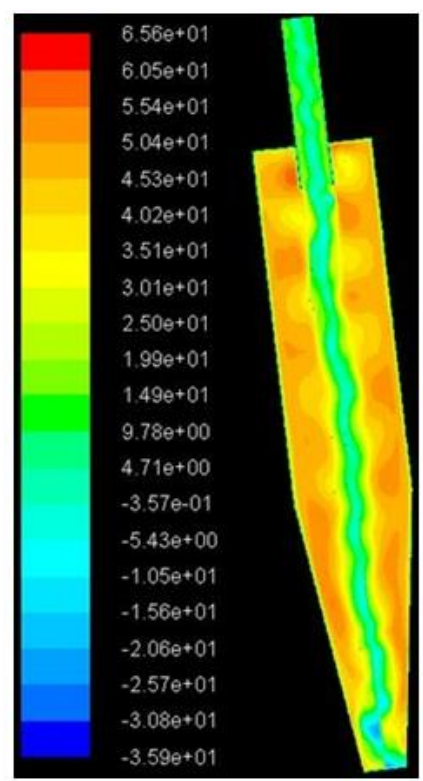

(a)

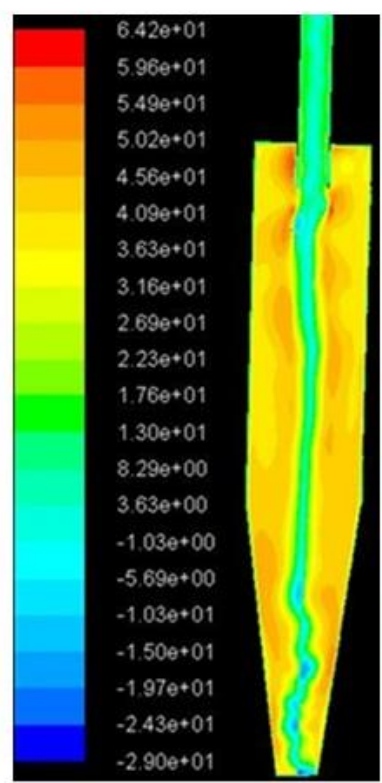

(b)

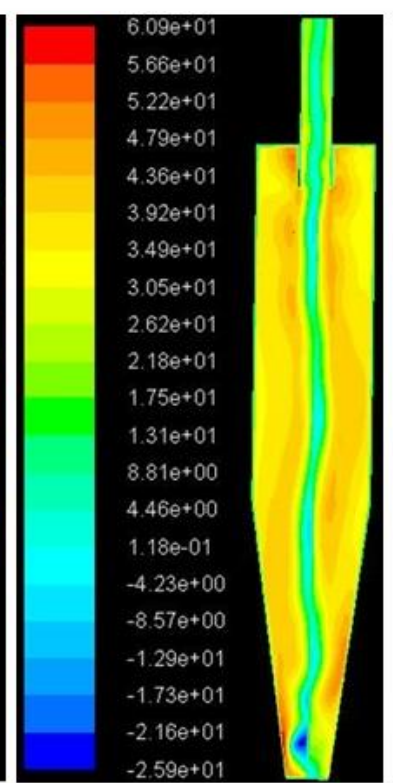

(c)

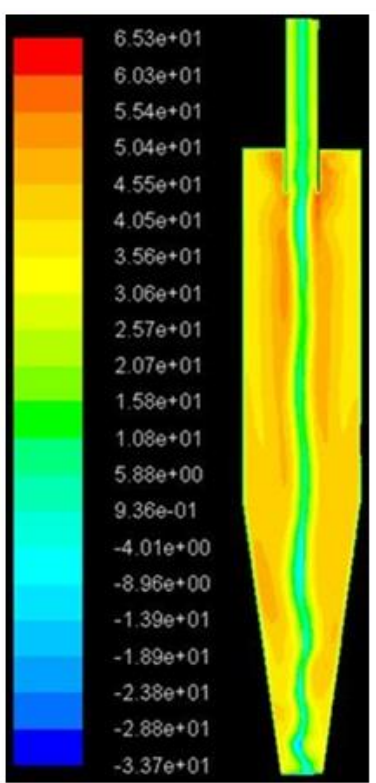

(d)

Figure 5.3: Tangential velocity contour for four cyclone bioaerosol geometry with the angle of (a) $30^{\circ}$, (b) $40^{\circ}$, (c) $45^{\circ}$ and (d) $60^{\circ}$ 


\subsubsection{Axial velocity}

In case of axial velocity, axial velocity near the cyclone wall leads to negative values and afterwards it becomes positive towards the cyclone center. As the gas flows into the vortex finder region, the highest axial velocity is obtained near the wall of a vortex finder region. It can be seen from figure 5.4 that the axial velocity near the sampler wall has negative values and it becomes positive towards the sampler center. Higher axial velocities are found at the center of the cylindrical section than in conical section. The outlet of the sampler has a negative axial velocity values in the whole cross-section. The changes of axial velocity in radial direction is largest than the changes in axial direction. The contours plots of axial velocity of the cyclone are presented in figure 5.4.

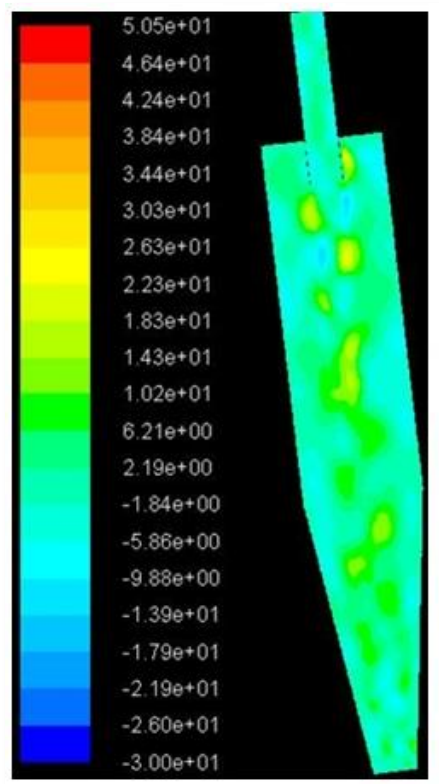

(a)

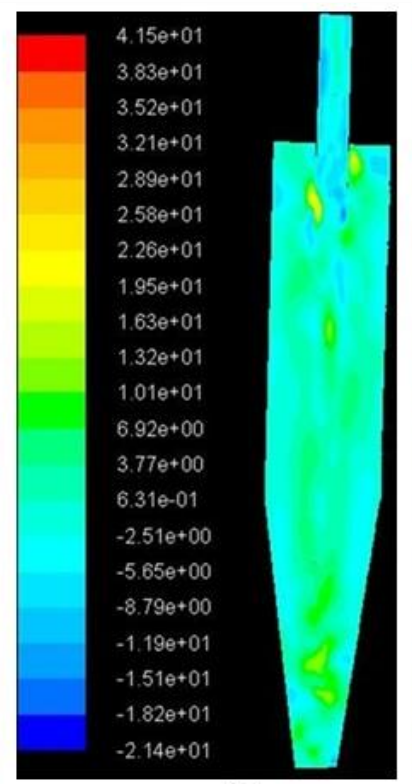

(b)

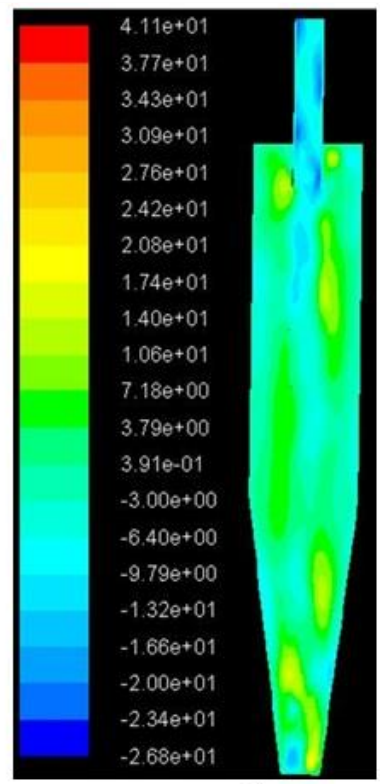

(c)

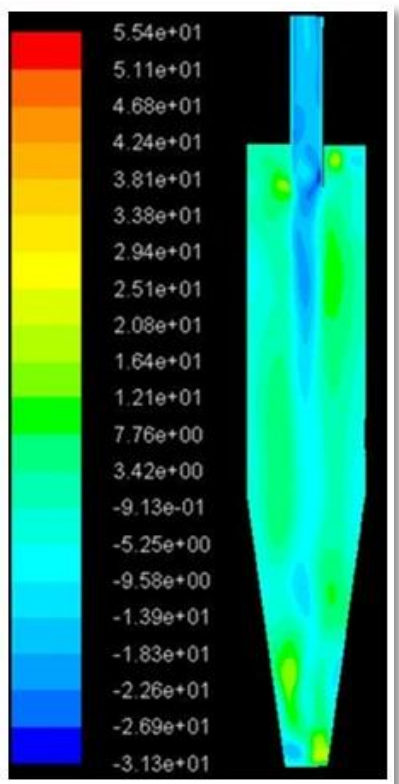

(d)

Figure 5.4: Axial velocity contour for four cyclone bioaerosol geometry with the angle of (a)

$$
30^{\circ} \text {, (b) } 40^{\circ} \text {, (c) } 45^{\circ} \text { and (d) } 60^{\circ}
$$


The variation of axial velocity with changing inlet tube angle is most likely the same on near to the wall, especially in the cylindrical part of the sampler. The change of axial velocity is more noticeable in the central section with the change of inlet angle.

\subsubsection{Radial velocity}

On the velocity distribution of the cyclone sampler, the radial velocity has a smaller magnitude while tangential and axial velocities always maintain a positive value. For the contour plot of the radial velocity, the radial velocity experienced some negative inward velocity as the flow enters into the rotating stream of the cyclone. The radial velocity also gained positive velocity because of the centrifugal force. Radial velocity becomes negative again around the vortex finder region as the flow directing to the center. Contours plots of radial velocity are shown in figure 5.5.

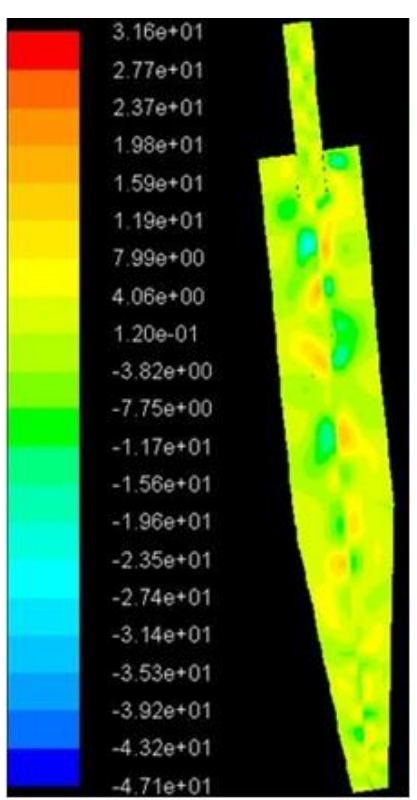

(a)

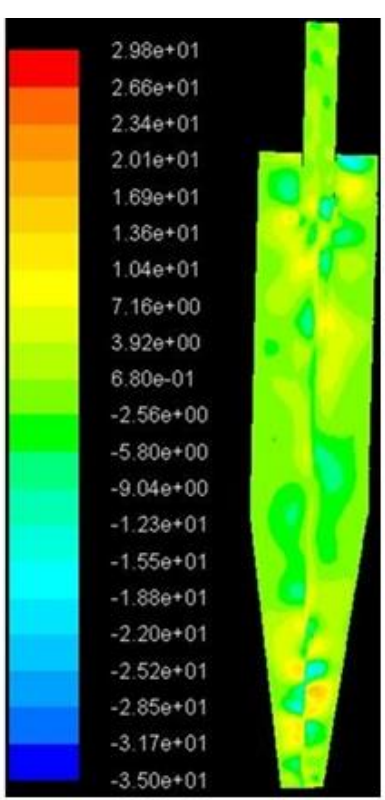

(b)

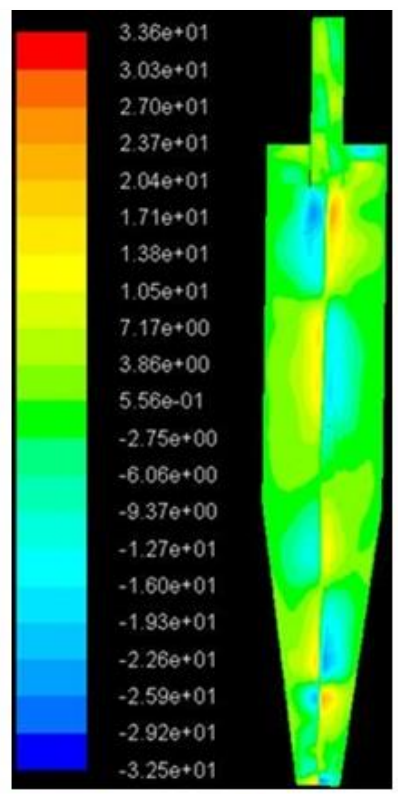

(c)

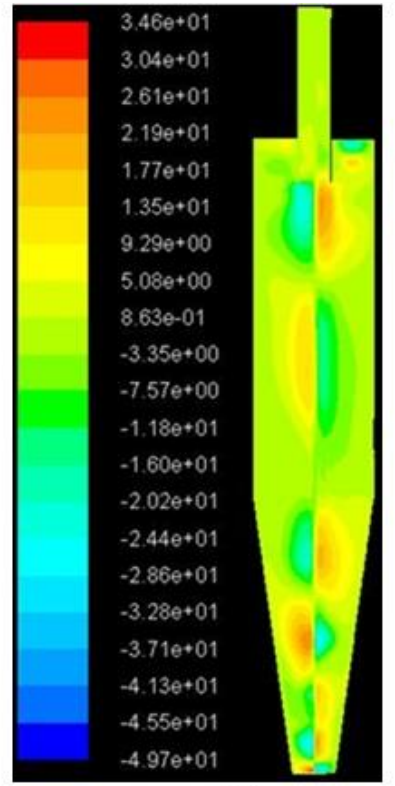

(d)

Figure 5.5: Radial velocity contours for cyclone bioaerosol geometry with an angle of (a) $30^{\circ}$, (b) $40^{\circ}$, (c) $45^{\circ}$ and (d) $60^{\circ}$ 
In the conical section, the radial velocity of upward flows show average magnitude and the maximum radial velocities are close to the inlet velocity. In this section, the velocity gradually shifts inward along the axial direction. In case of cylindrical section, the intersection point between upward flows and downward flows is independent of the axial position.

\subsection{RESULTS OF DISCRETE PHASE SIMULATION ON CYCLONE SAMPLER}

Because particle phase characteristics cannot be easily described by continuum models, Lagrangian approach is used to define the particle phase. Once the continuous phase model was determined, the Discrete Phase Model (DPM) was used to resolve for the particles trajectories of different range. Since all the particles being considered in this thesis were in the micron range, their effect on the fluid during particle trajectories calculation can be neglected. The particle motion can be expressed by differential equations in Lagrangian reference frame and incorporated to get individual particle tracking [58]. After obtaining the dynamic behavior of the gas phase by Eulerian approach, Lagrangian-equation for a particular moving particle is to be solved. As particle velocity and particle trajectory are computed for each particle, this approach is more appropriate to get the discrete motion of particles. Flow behavior is reviewed in the terms of velocity components of the sampler.

\subsubsection{Particle residence time}

Figure 5.6 shows the particle residence time tracked by the dispersed particle of the system. The calculation of the residence time begins when a particle of the cyclone barge into the system and terminates when the particle exits. Particle residence time is used to predict the time during which a particle remains within the system. A longer residence time will results on higher particle collection efficiency which is actually an important parameter in particle-fluid flow of 
cyclone sampler. Distribution of particle residence times in the cyclone sampler can be estimated by the interchange of particles between the down flow and core regions.

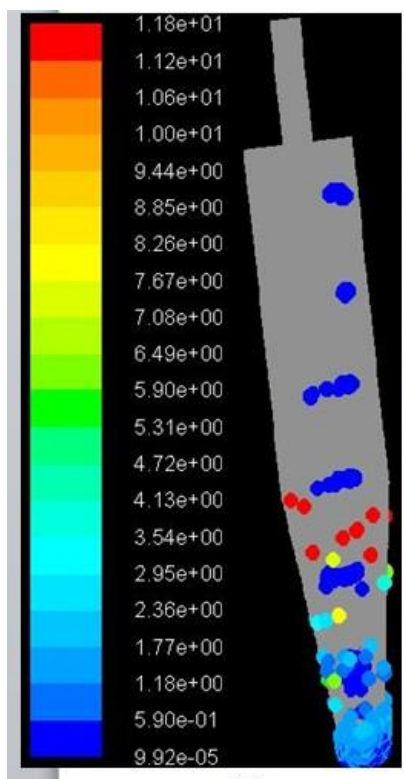

(a)

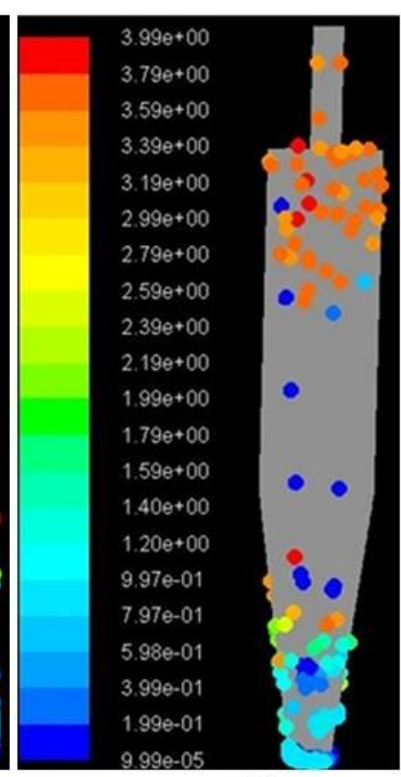

(b)

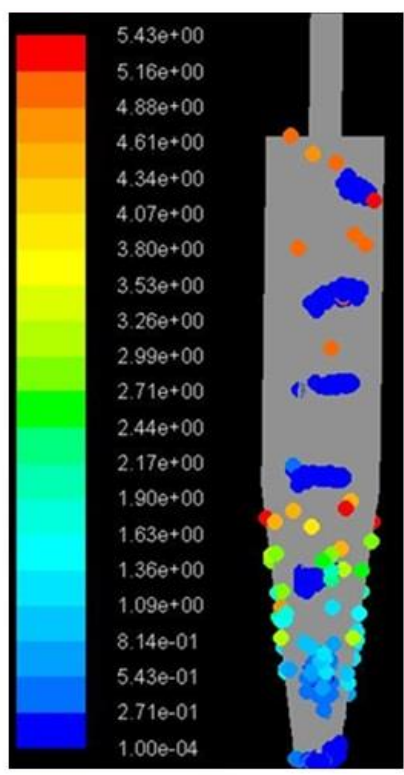

(c)

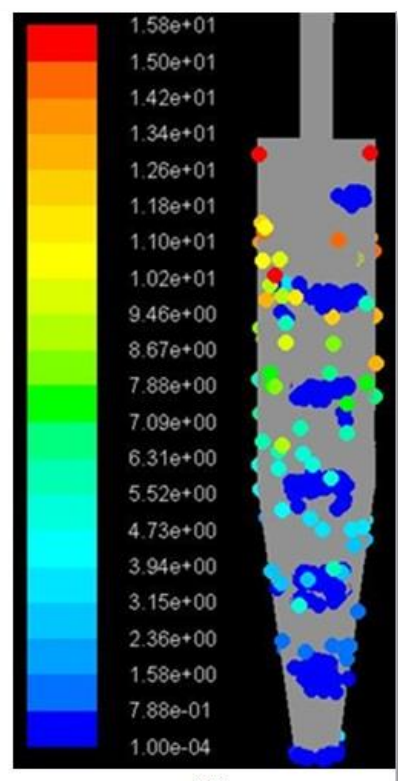

(d)

Figure 5.6: Trajectories of particle residence time for cyclone bioaerosol geometry with the angle

$$
\text { of (a) } 30^{\circ} \text {, (b) } 40^{\circ} \text {, (c) } 45^{\circ} \text { and (d) } 60^{\circ}
$$

The red color indicates that those particles stayed longer than any other particle of the system and then took exit through the outlet. Blue color signifies that those particles took less time to stay on the system and may collect on the system. Residence time is dependent on the inflow and outflow characteristics. With the variation of inlet and outlet dimensions, the residence time may vary. The effects of inlet angle have an influence on the particle residence time. For inlet section angle of $60^{\circ}$, it can be said that more particle are collected on the receptacle which is signified by their color. These particles did not stay longer on the cyclone and eventually deposited at the bottom of the sampler. While for $40^{\circ}$ inlet angle all of the particle exit through the outlet; most of the particles retained onto the tube for inlet angle of $30^{\circ}$. So, the residence time varies according to the inlet tube angle. 


\subsubsection{Particle velocity magnitude}

Particle traces colored by the velocity component of the particle are shown in Figure 5.7. When the particle enters into the system, they have minimal initial velocity.

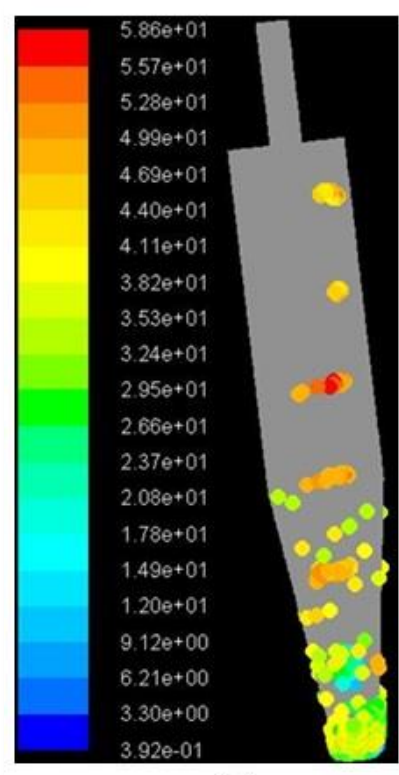

(a)

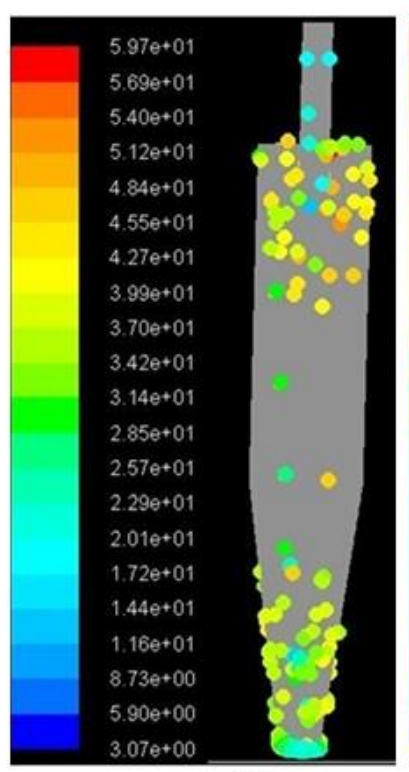

(b)

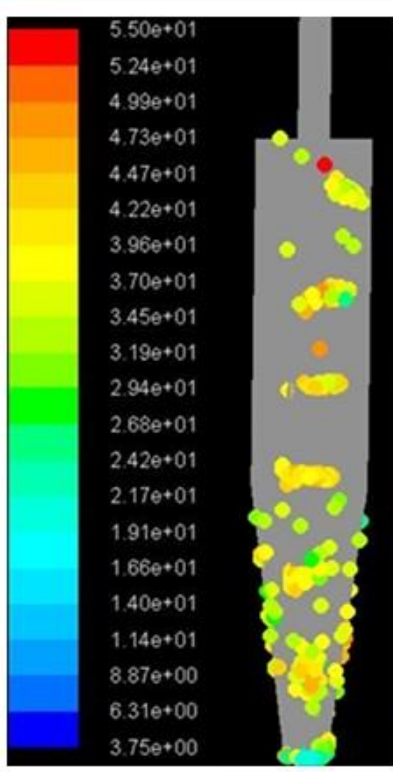

(c)

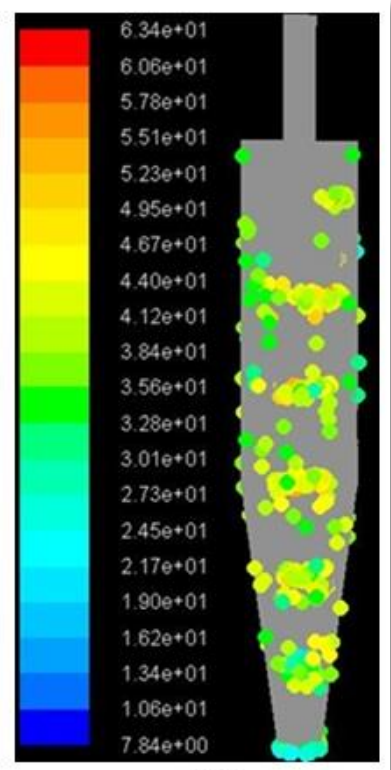

(d)

Figure 5.7: Trajectories of particle velocity magnitude for cyclone bioaerosol geometry with the angle of (a) $30^{\circ}$, (b) $40^{\circ}$, (c) $45^{\circ}$ and (d) $60^{\circ}$

When a particle enters into the cyclone outer vortex with the air stream, a radial acceleration occurs due to the centrifugal force acting on the particle. A different particle and air stream path can be generated by the relative velocity between the particle and air stream. When the particles arrive near the walls, they are moved down the sides of the walls to the bottom end of the cyclone sampler. As they move to the bottom by rotating through the centrifugal effect of the system, their velocity may increase and may reach to their maximum velocity. Particle velocity is not influenced by the neighboring particles. The fluid pattern of the system is not changed by the relative velocity between the air stream and particle. Higher particle concentration value can be achieved by the gathering of particles in a specified region. Velocity 
magnitude on discrete phase for cyclone sampler with four different geometry show identical pattern.

\subsubsection{Particle y velocity magnitude}

Particle traces colored by the vertical velocity component of the particle are shown in figure 5.8.

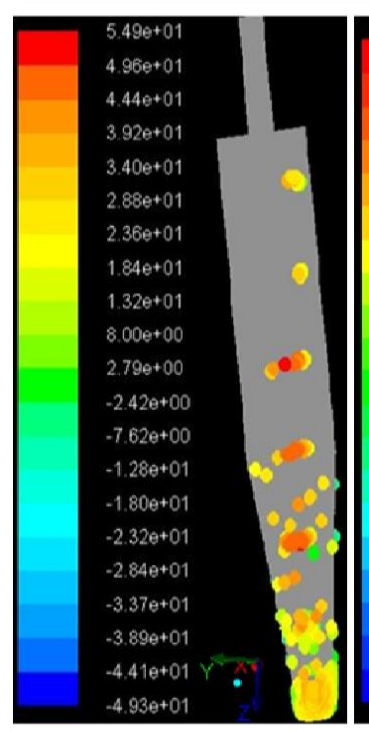

(a)

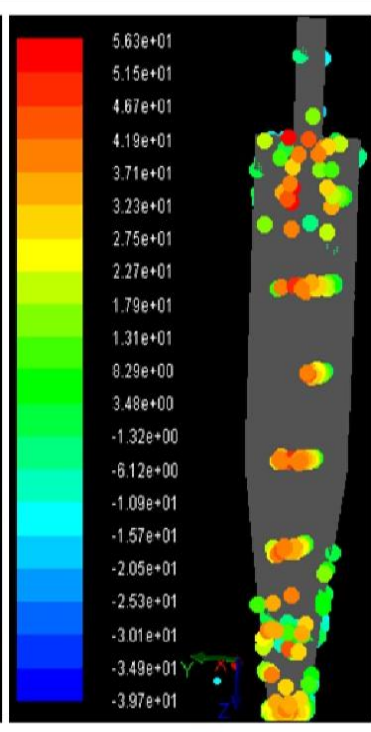

(b)

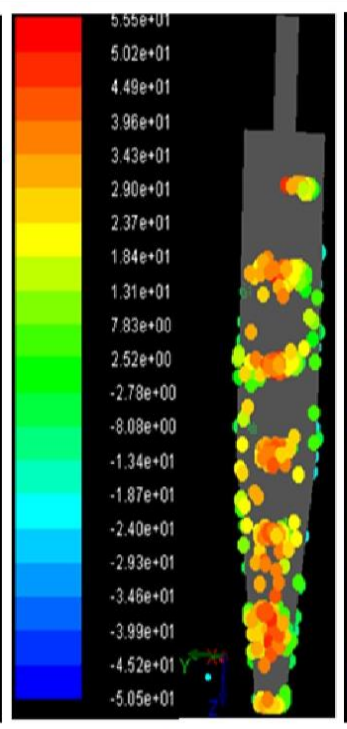

(c)

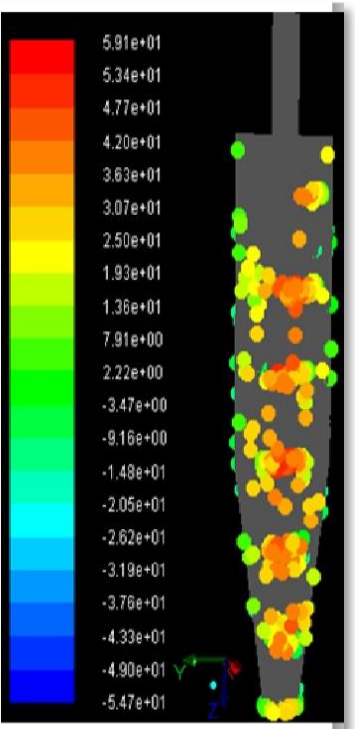

(d)

Figure 5.8: Trajectories of particle y velocity magnitude for cyclone bioaerosol geometry with the angle of (a) $30^{\circ}$, (b) $40^{\circ}$, (c) $45^{\circ}$ and (d) $60^{\circ}$

When a particle moves from one space to another, then there is a change of their vertical velocity component. Particles moves downward near the cyclone wall and upward near the sampler axis. This downward particle velocity near the wall causes the particle movement from the sampler wall to the bottom of the sampler. This flow pattern is created by the double vortex flow generation, which centrifuges the particle onto the walls. If a particle is flowed out with a vertical velocity, it will be decelerated gradually. The vertical deceleration magnitude will be greater, however, the greater the vertical velocity relative to the fluid. The magnitude of the 
vertical velocity component is shown in figure 5.8 which shows a higher magnitude for all of the four samplers. It can be seen from the above figure that, particle y velocity is nearly identical for these cyclone samplers. Outer vortex flow largely depends on the inner wall smoothness. Rough surfaces effects in a turbulent flow regime which tends to increase the particle re-entrainment.

\subsection{Streamline Pattern for CyClone SAMPler}

Figure 5.9 shows the streamline of the cyclone sampler for velocity using ANSYS-CFX. The $\mathrm{X}, \mathrm{Y}$ and $\mathrm{Z}$ axes of the cyclone sampler are also shown through the figure 5.9. Particles enter through the inlet with the maximum inlet velocity and a strong vortex created inside the cyclone due to centrifugal force. Then particles which have sufficient inertia, strike onto the cyclone wall and these particles are either retain on the tube or move to the bottom of cyclone due to the secondary flow created in the sampler. In case of most centrifugal-based cyclone sampler designs, the end of the vortex approbated to stroll around the lower walls of the sampler. This end has an influential effect on the collection efficiency, clogging and wear of cyclones due to its position and several characteristic qualities. As the flow is moving from outer to inner vortex region, an inward drag force is acting on the particle which is proportional to particle diameter. Thus larger particles are separated from the particle-laden flow and smaller ones entrapped into inner vortex and then escape from the sampler the sampler. 


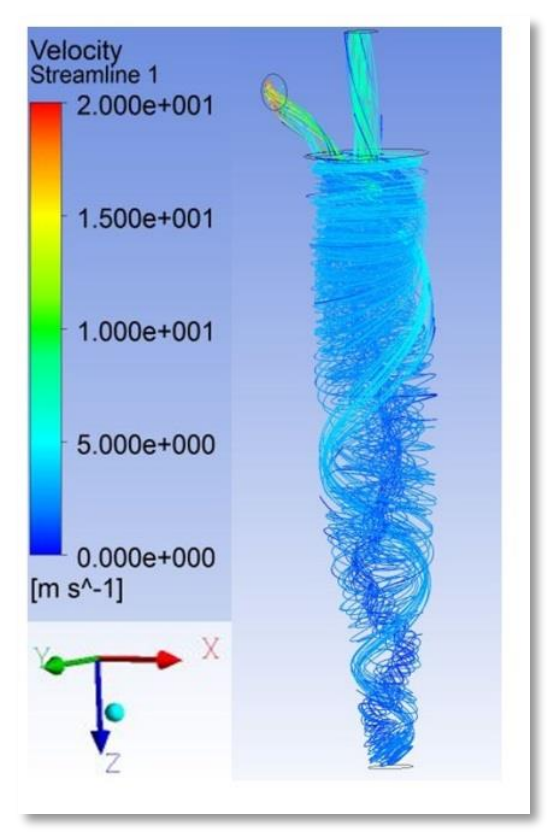

Figure 5.9: Streamline of the cyclone sampler for velocity function with the angle of $40^{\circ}$

\subsection{DIMENSIONLESS VELOCITY PROFILES MEASUREMENT}

Dimensionless tangential velocity profiles were measured along the $\mathrm{x}$ - axis at different stations of the cyclone sampler. The mean tangential velocity profiles were calculated at different stations from $z / D=0.5, z / D=1$ to $z / D=2$ along the $x$-axis, as the tip of the vortex finder was taken as $z / D=0$. Figure 5.10 to 5.12 shows the measured mean tangential velocity profiles at three different stations of the cyclone sampler. This type of velocity distribution shows a Rankine-vortex structure with good symmetry in case of a strongly swirl-dominated flow. The measurement tangential velocities show good agreement with the results. The position and the value of the maximum tangential velocities are not dependent on the axial positions which indicate that swirl does not weaken in the separation space. 


\subsubsection{Tangential velocity profiles at $\mathrm{z} / \mathrm{D}=\mathbf{0 . 5}$}

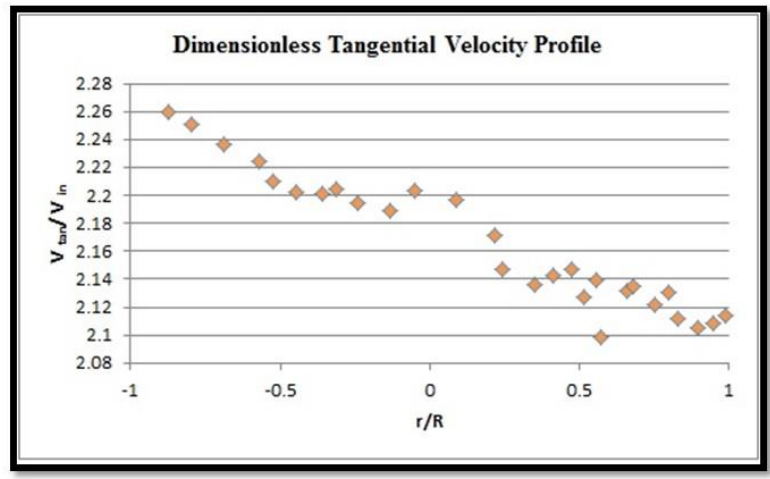

(a)

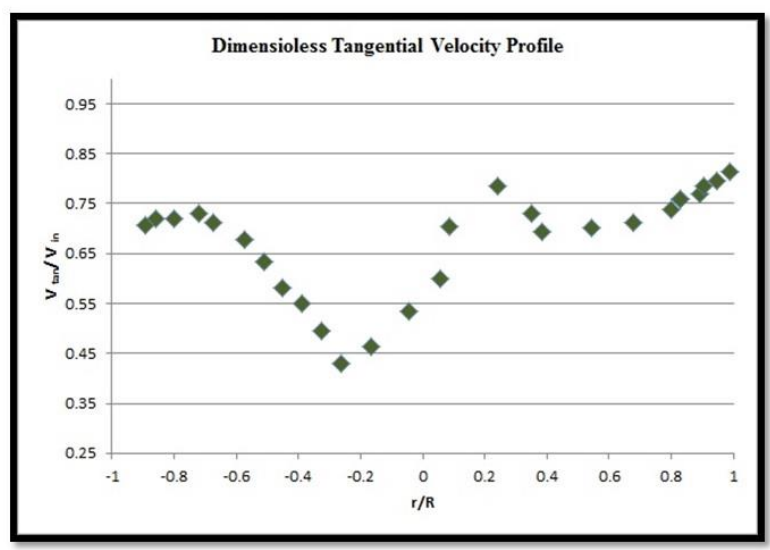

(c)

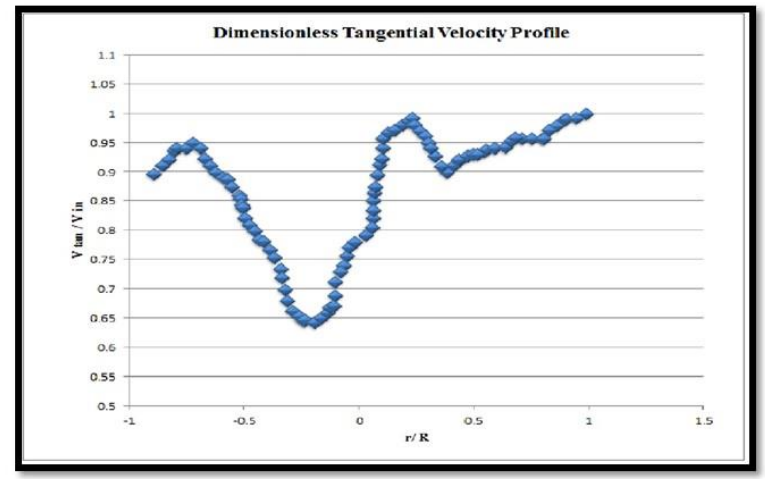

(b)

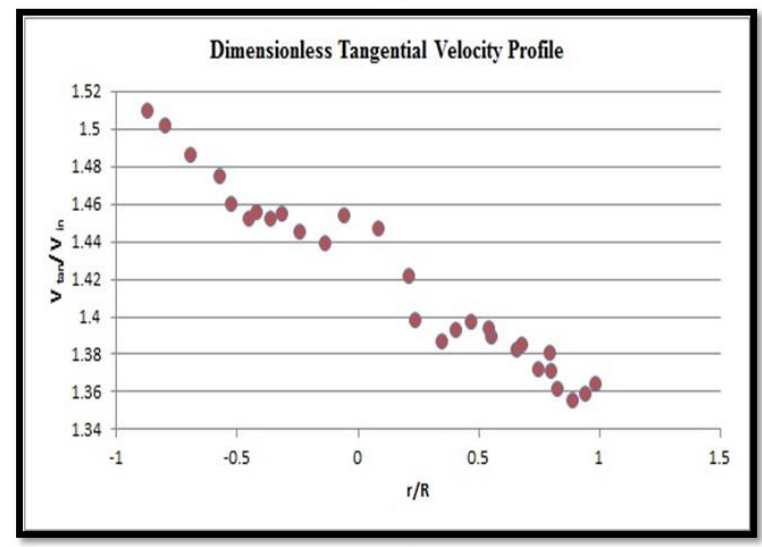

(d)

Figure 5.10: The mean tangential velocity profiles for cyclone sampler at $\mathrm{z} / \mathrm{D}=0.5$ for different inlet tube angle (a) $30^{\circ}$, (b) $40^{\circ}$, (c) $45^{\circ}$ and (d) $60^{\circ}$

Figure 5.10 illustrates the tangential velocity profiles for four cyclone sampler with different inlet angles respectively. It can be seen that the shapes of tangential velocity profiles are not very different in case of inner upward and outer downward flow. The boundary of these flows gradually moves outward along their axial directions. With the increase of velocity component along radial directions, this boundary changes their positions. The magnitudes of the tangential velocity in inner and outer flow regions decrease at first then they gradually increase with their centrifugal effects in figure 5.10 (b) and (c). Afterwards, they somewhat fluctuates with their radial positions. 
High speed gas enters into the system with a pure radial flow and then the velocity decreases as the gas spins down. Since the gas flow strikes with the secondary flow of the system, a chaotic flow is observed on this position. For figure 5.10 (a) and (d), gas velocity decreases sharply at the outside surface of the vortex finder, which results lower collection efficiency. Comparing the tangential velocity profile from the above figure, the flow field shows an identical pattern among all of these cyclone samplers.

\subsubsection{Tangential velocity profiles at $\mathrm{z} / \mathrm{D}=1.0$}

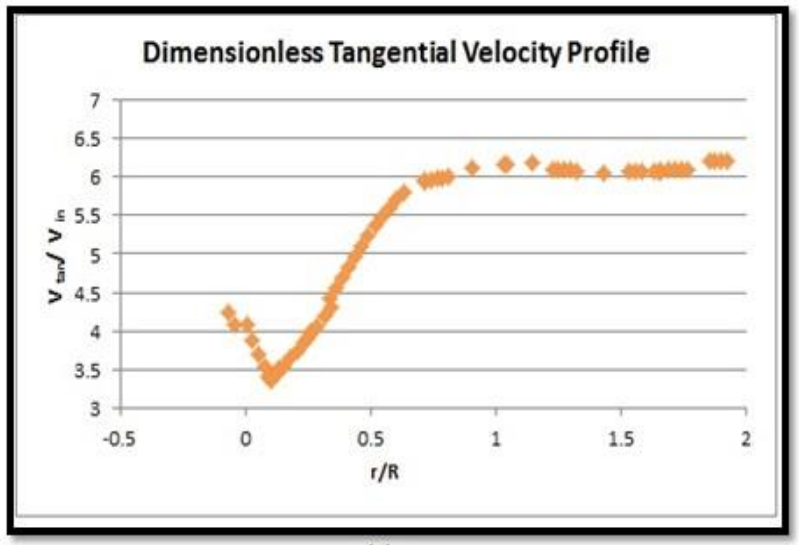

(a)

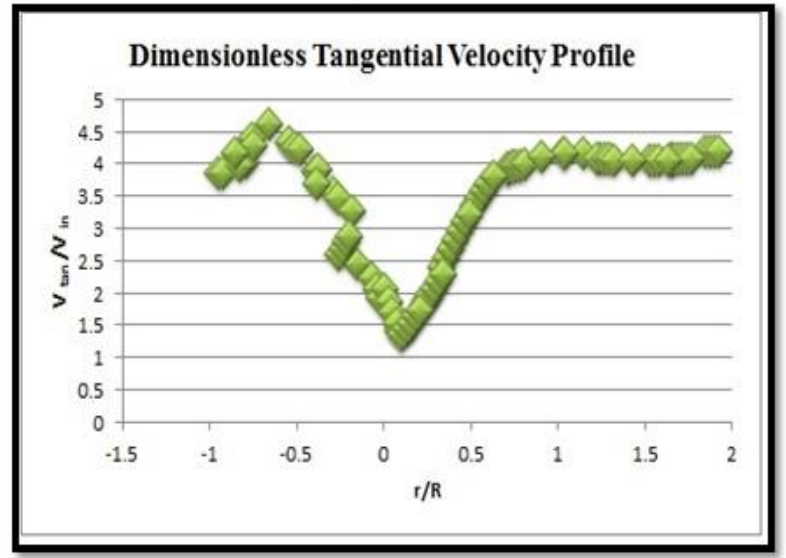

(c)

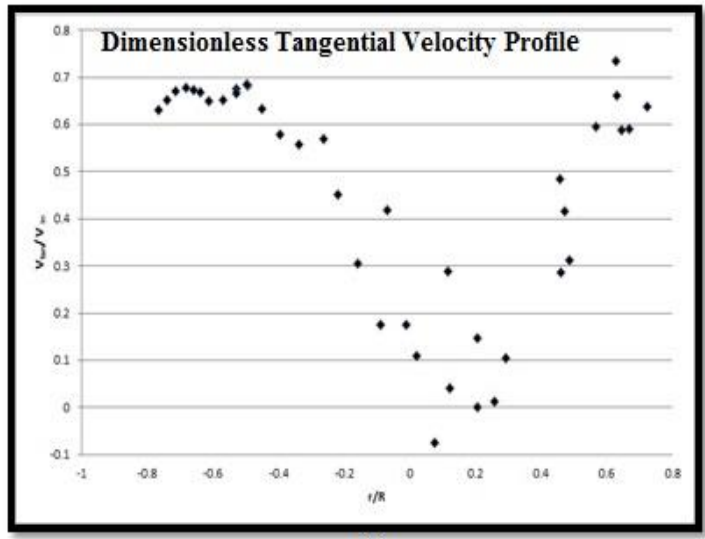

(b)

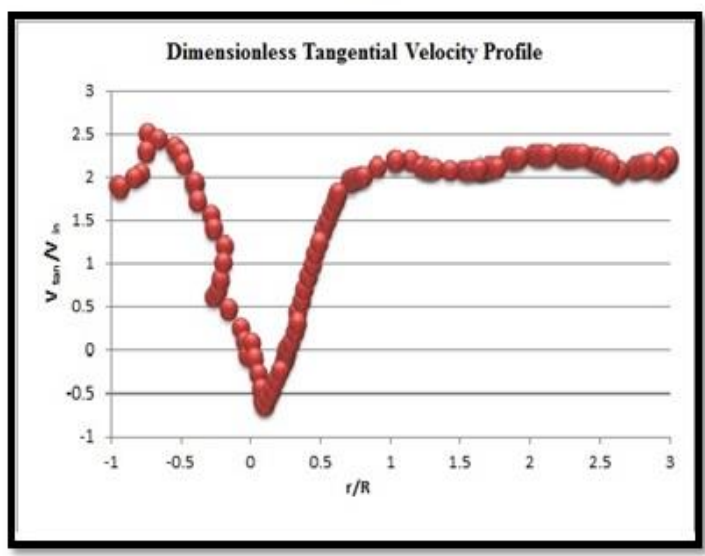

(d)

Figure 5.11: The mean tangential velocity profiles for cyclone sampler at $\mathrm{z} / \mathrm{D}=1.0$ for different inlet tube angle (a) $30^{\circ}$, (b) $40^{\circ}$, (c) $45^{\circ}$ and (d) $60^{\circ}$ 
Figure 5.11 shows the variation of tangential velocity profiles at $\mathrm{z} / \mathrm{D}=1.0$ for different inlet tube angles of four cyclone samplers. Velocity distribution in a cyclone sampler is a complex phenomenon, as tangential velocity varies with the circumferential, radial and axial directions of the sampler. It also defines the centrifugal force applied to the air stream and to the particles. Tangential velocity profiles at $\mathrm{z} / \mathrm{D}=1.0$ position display Rankine-vortex pattern for all the four samplers. The main flow of the cyclone comprised of an outer spiral flow which moves downward from the sampler inlet and an inner spiral of smaller radius flow which moves upward into the exit tube. The outer spiral downward moving flow occurs at outer vortex region and an inner spiral upward moving flow visible in inner vortex region. The inner and outer vortex regions show a significant change with their radial positions. In the outer vortex region, the tangential velocity increases with a decrease of the rotational radius of the cyclone sampler. At the boundary of the outer vortex and inner vortex region, this velocity increases to its maximum magnitude. In the inner vortex, the tangential velocity decreases with the decrease of rotational radius. It can be noted that the fluid flow starts from the bottom of the exit tube from the outer vortex to the inner one and moves down into the conical part at the bottom of the cyclone sampler. The maximum tangential velocity decreases progressively along the downward axial directions. It is clearly seen from this figure that tangential velocity profiles show a similar pattern for all these cyclone samplers.

\subsubsection{Tangential velocity profiles at $\mathrm{z} / \mathrm{D}=2$}

A better representation of the results for four different cyclone samplers can be seen in terms of their tangential velocity distribution as shown in these above figures. Data obtained from the RSM turbulence model to signify the radial distributions of tangential velocity on the cyclone sampler are presented in figure 5.10 to 5.12 , in which the effect of inlet angle on flow 
field inside the cyclone is verified. The high tangential velocities can be found near the cyclone wall region. Velocities gradients across vertical axes can be observed while large eddies on reverse flow region are seen also. The tangential velocity profiles were drawn from the obtained data of this study at three different stations along $\mathrm{x}$-axis of the sampler.

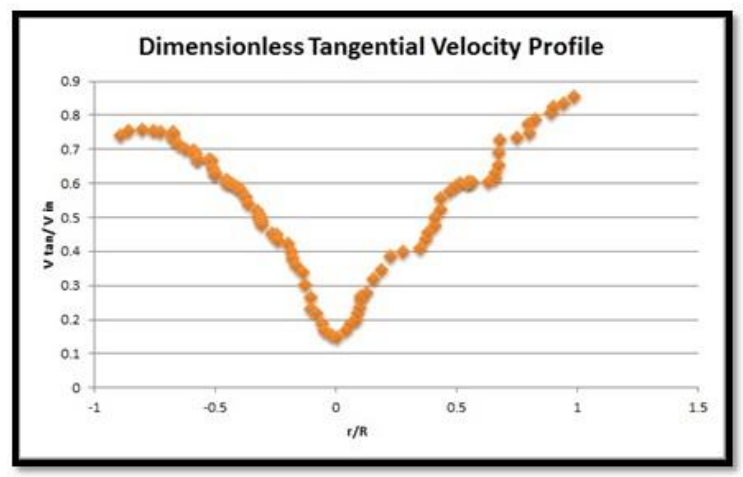

(a)

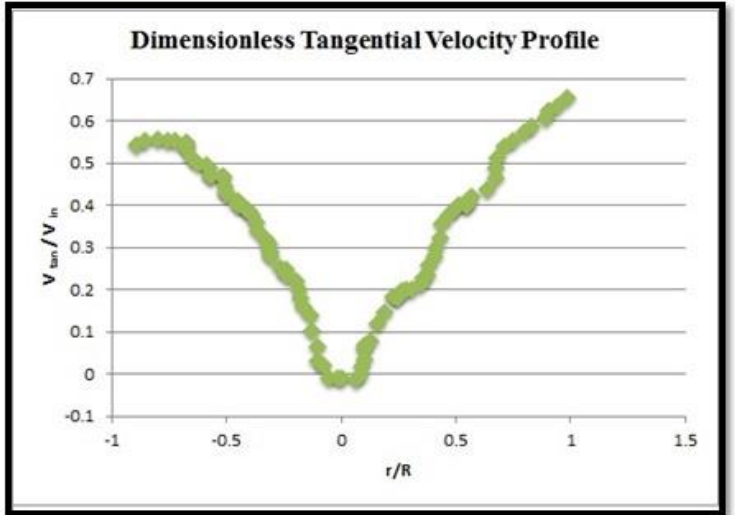

(c)

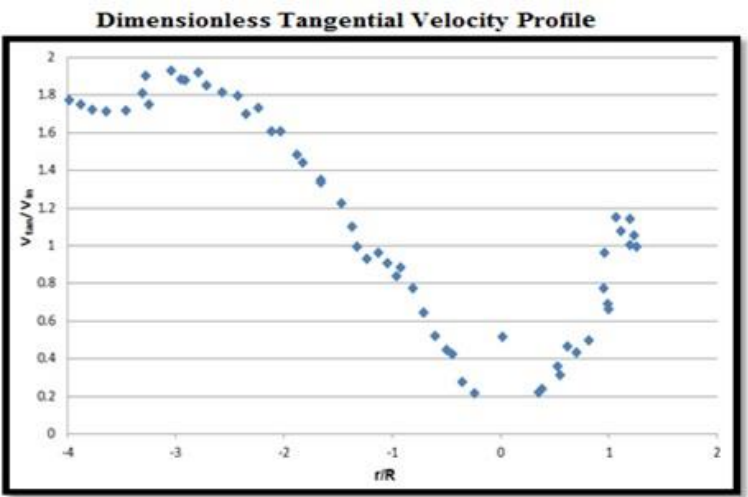

(b)

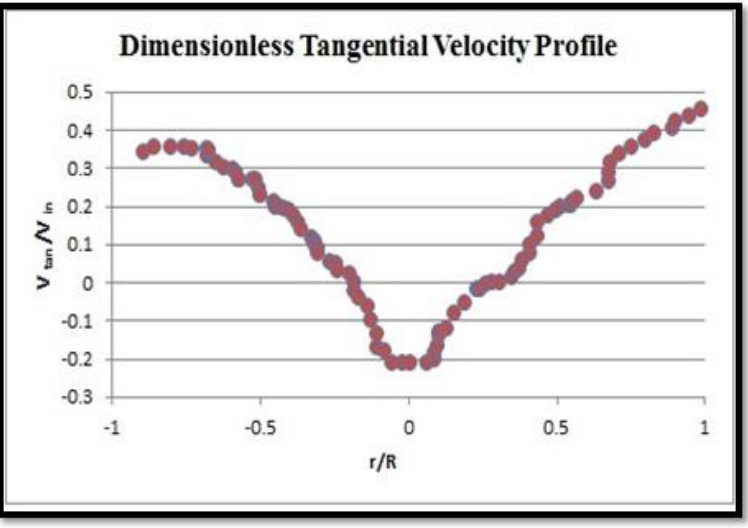

(d)

Figure 5.12: The mean tangential velocity profiles for cyclone sampler at $\mathrm{z} / \mathrm{D}=2.0$ of different inlet tube angle (a) $30^{\circ}$, (b) $40^{\circ}$, (c) $45^{\circ}$ and (d) $60^{\circ}$

Figure 5.12 shows the mean tangential velocity profiles at $\mathrm{z} / \mathrm{D}=2.0$ of the cyclone sampler. The tangential velocity distribution comprises of an upward-flow zone and a downward-flow zone with good symmetry. The magnitude of the tangential velocity is almost in same order of the inlet velocity in the upward-flow region and smaller than the inlet velocity by 
an order of magnitude in the downward-flow region. The recirculation occurs near the axis and the inlet of the vortex finder which may results to the average pressure increment in the vortex finder. With the increase of axial position, tangential velocity gradually shifts inward along the axial direction and displays a cone shape.

From the above figures, it was found that with the increase of the radial position of the sampler, the tangential velocity increases. So tangential velocity component at $\mathrm{z} / \mathrm{D}=2$ position shows similar pattern, while velocity component at $\mathrm{z} / \mathrm{D}=0.5$ exhibits some fluctuations. It can be seen from these figures that both the upward and the downward velocities are increasing with inlet velocity of the sampler. Low velocities are visible in the core of the rotating flow, which is adjacent with the center of the cyclone sampler (not in coincidence). As the distance from the core is increasing, the velocity is accrescent to a peak value and then decreases again. For the cyclone with $30^{\circ}$ inlet angles have higher values than the cyclone with $60^{\circ}$ inlet tube angle in most cases. The tangential velocity distribution in each cross-section shows a symmetric pattern, gradually alternates from higher to lower velocity, and then shifts towards high velocity again. This pattern is clearly visible in all the above numerical results. High velocity fluctuation is

found near the center of the cyclone due to the advancement of the vortex core region. As the fluid around the wall moves to underflow, the downward velocity increases consecutively. The fluid moves then towards outlet and the upward velocity is getting increase with its radial distance.

\subsection{Velocity vectors for cyclone sampler}

Velocity vector analysis for the velocity distribution is shown in the figure 5.13 . Velocity 
profile in vector plot for cyclone inclined of $45^{\circ}$ inlet angle shows the top portion, bottom portion and center portion of the sampler with recirculation zones respectively. Fig. 5.13 (a) displays the top section of the cyclone sampler which shows the inlet and outlet velocity vector of the sampler. Velocity vectors are influenced by the flow rates in the cyclone sampler. Velocity vectors near the inlet and outlet segment exhibits the flow field in this region. Particles enter into the sampler with a high velocity through the inlet and gradually decrease as it moves in radially to the downward portion. Then it advances some momentum at hindmost portion of the inlet. In Fig. 5.13 (b), Air flows in a spiral pattern from the top to the bottom of the cyclone and particles in the flow are subjected to centrifugal forces because of the creation of a strong vortex inside the cyclone sampler. Fig. 5.13 (c) shows the velocity vector along the center-plane which intersects the whole cyclone sampler vertically.

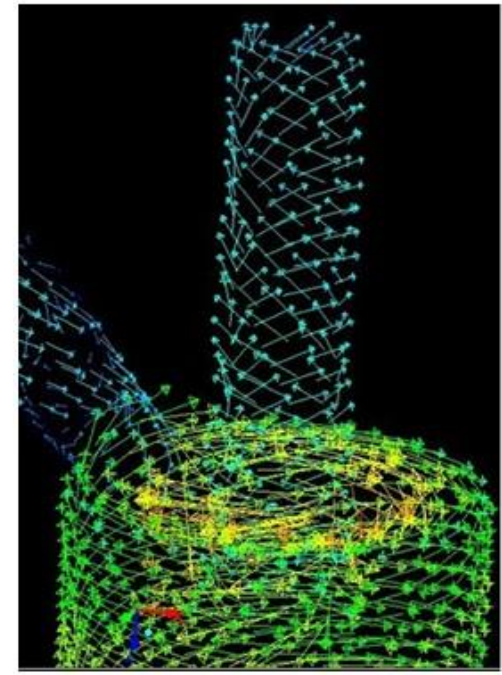

(a)

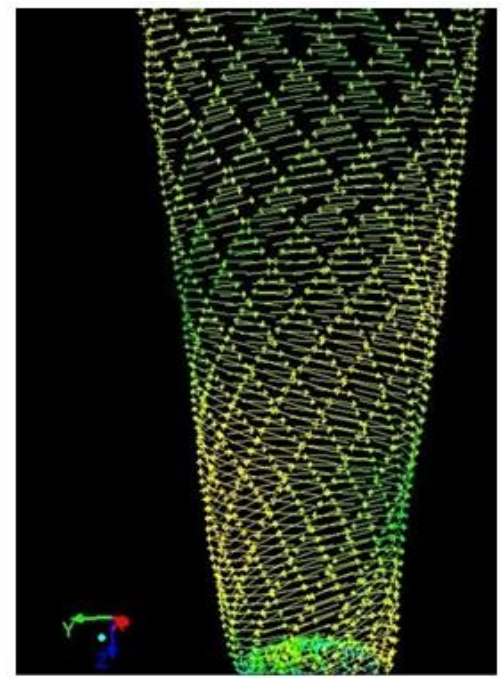

(b)

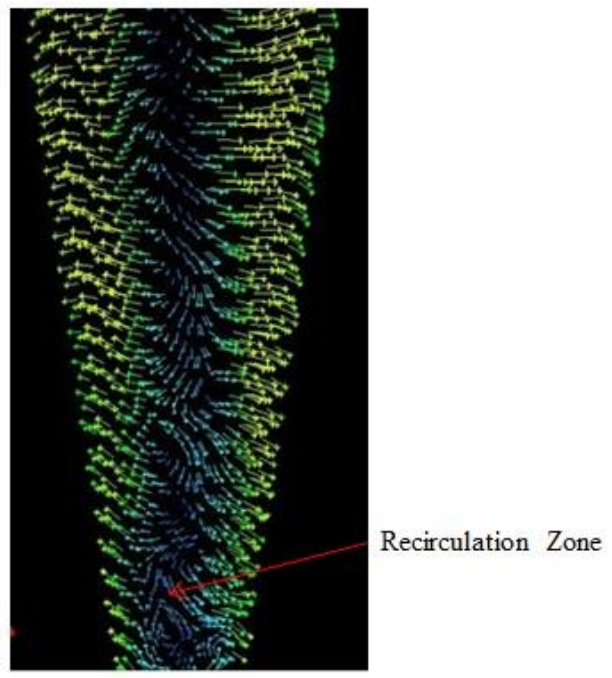

(c)

Figure 5.13: Velocity profile in vector plot for cyclone inclined of $45^{\circ}$ inlet with (a) top section, (b) bottom section and (c) slice of cyclone sampler with recirculation zone

The velocity vector plot shows some of the minor flow patterns in the sampler flow field. A recirculation zone appears beneath the vortex finder region, which is clearly visible at the 
downward portion along the cyclone wall and the direction of the flow turns over along the bottom part of the conical section. The solid recirculation seems did not to have an effect in these areas. Fig. 5.13 shows the vectors details in terms of velocity profiles for the cyclone sampler where a low centrifugal force is observed near the above of the conical section of the cyclone.

\subsection{FLOW PATTERN ON DIFFERENT PLANE OF CYCLONE SAMPLER}

Figure 5.13 represents typical flow patterns at the inner portion as well as at different planes (xz) of a cyclone sampler. Two parallel swirling flow patterns are visible from this figure i.e., one is near the wall and other one is the secondary one which shifts towards the over flow outlet section. Since high swirling velocity is observed at the inside of the cyclone sampler, a very low velocity zone is created at the central region. The whole central low velocity zone signifies a definite air core formation in the cyclone sampler. An air core region is established in cyclone sampler because the swirling flow produces a negative gauge pressure region along the axis. The velocity vectors shows upwards flow pattern towards the vortex, near the outlet portion.

Figure 5.13(b) illustrates the flow pattern at the conical part of the sampler. The velocity vector shows that the upward fluid flow is moving near the central air core with a very fast velocity. High swirling flow is perceptible on both inner and outer sides of the cyclone. Then the swirl actions are reversed between the outer and inner zones, when the flow moves towards the outlet section. A recirculation zone is clearly visible through the velocity vector plot. The fluid flow is moving downward along the sampler wall and reverses along the lower part of the conical section. 


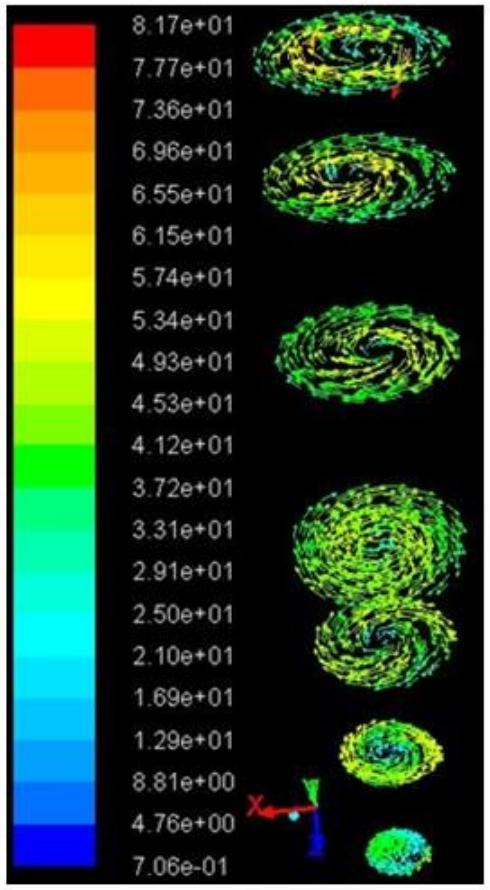

(a)

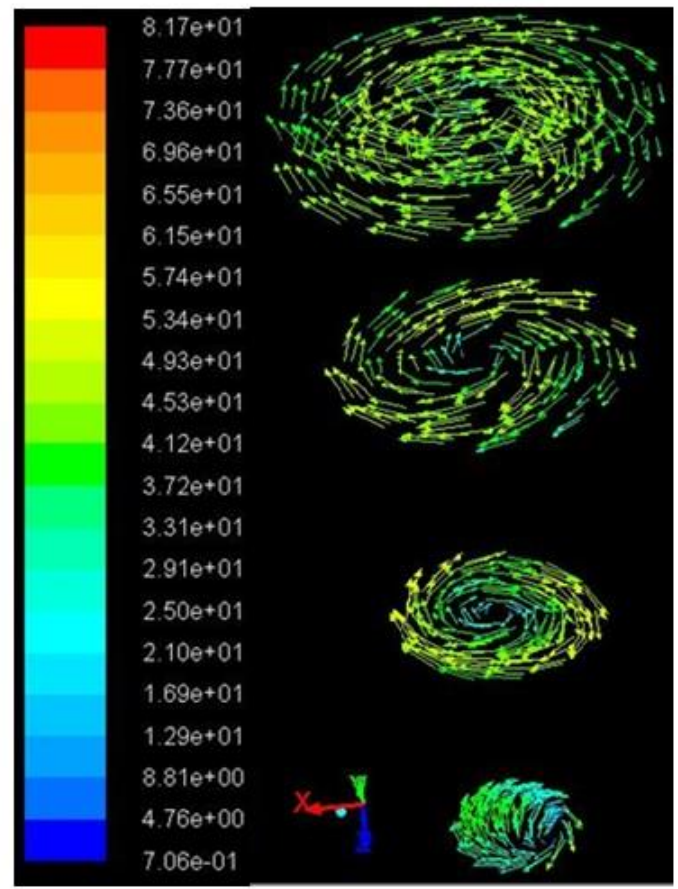

(b)

Figure 5.14: Velocity vector patterns in different planes of cyclone inclined of $45^{\circ}$ inlet with (a) different plane from top to bottom section, (b) planes at conical section

\subsection{COLLECTION EFFICIENCY CALCULATION}

The calculation of the collection efficiency of the particle is followed by the equation 4.2 and 4.3 used on this literature. In case of overall collection efficiency calculation, which is based on the following equation and suggested in these literature [59] [60] [61].

Overall Efficiency $(\%)=\left[\frac{m_{\text {Underflow }}-m_{\text {Outlet }}}{m_{\text {Inlet }}}\right] * 100$

where, $m_{\text {Underflow }}=$ flow of collected solids, $m_{\text {Outlet }}=$ solid flow at gas outlet, $m_{\text {Inlet }}=$ solid flow at inlet cyclone. 


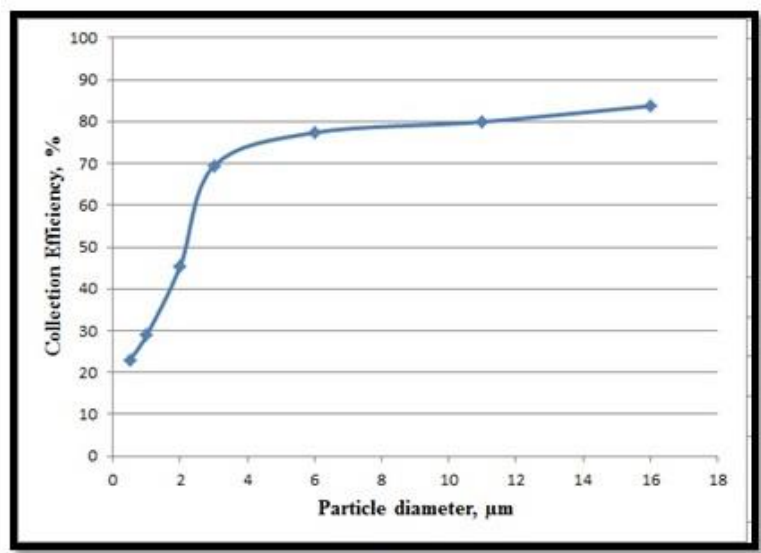

(a)

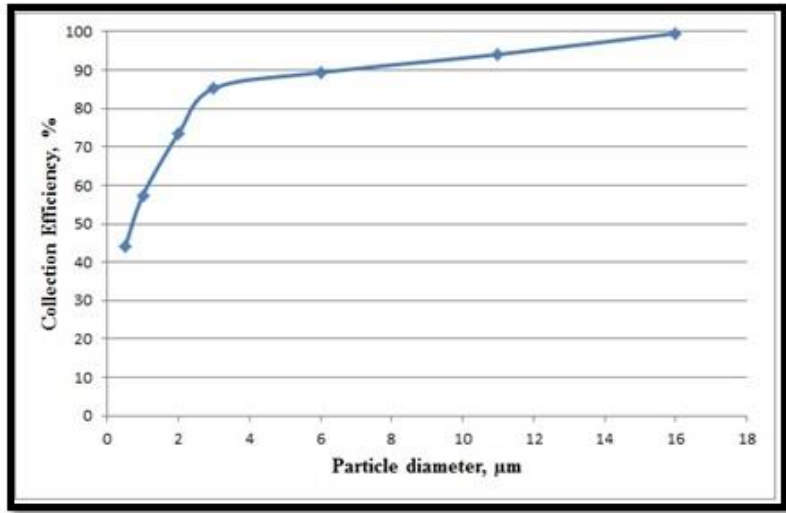

(c)

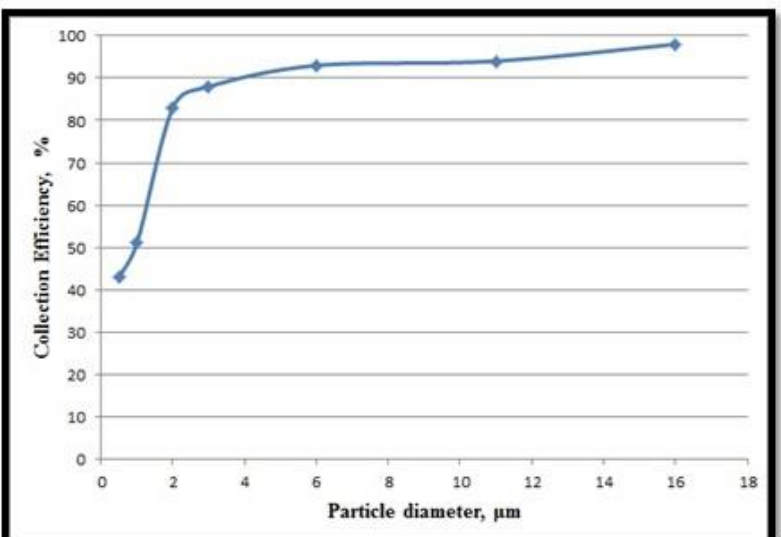

(b)

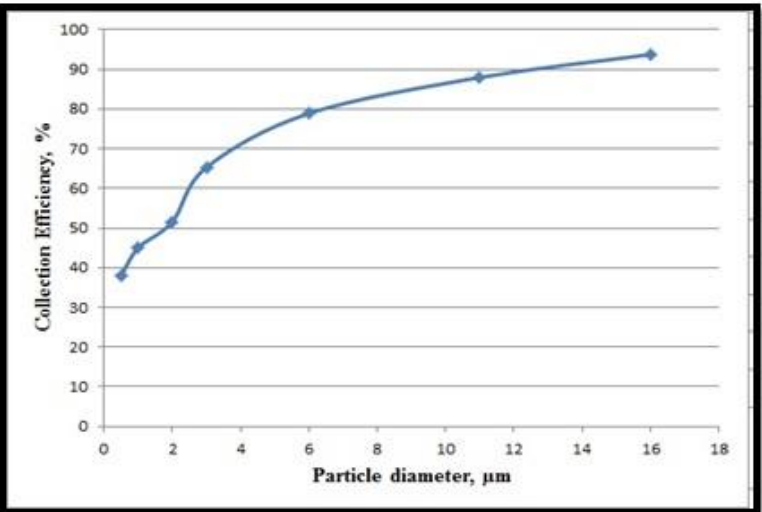

(d)

Figure 5.15: Collection efficiency curve with the particle diameter at flow rate of $\mathrm{Q}=4 \mathrm{~L} / \mathrm{min}$ for cyclone sampler of different inlet tube angle (a) $30^{\circ}$, (b) $40^{\circ}$, (c) $45^{\circ}$ and (d) $60^{\circ}$

Figure 5.15 represents the collection efficiency curve of four cyclone samplers at a flow rate of $\mathrm{Q}=4 \mathrm{~L} / \mathrm{min}$. It can be deduced from the above figure that the measured collection efficiency curve shows the ' $S$ ' shape pattern and goes well with the automated scheme used for analyzing dispersed phase equations. Collection efficiency of the sampler increases with the particle size. Particles with $2 \mu \mathrm{m}$ diameter and above retained more in the cyclone. As the size of the particle diameter increases, the higher collection efficiency is obtained in the sampler. So, the curve shifts down to the right with the greater value of the particle size. For the particle size of $16 \mu \mathrm{m}$, the collection efficiency obtained was $99.94 \%$. 
Table 5.1: Collection efficiency data studied for this project

\begin{tabular}{|c|c|}
\hline Cyclone inlet type & Collection efficiency (\%) \\
\hline $30^{\circ}$ angle & 83.51 \\
\hline $40^{\circ}$ angle & 95.58 \\
\hline $45^{\circ}$ angle & 99.94 \\
\hline $60^{\circ}$ angle & 92.02 \\
\hline
\end{tabular}

The numerical results presented in this study exhibits that RSM is able to demonstrate the effect of different cyclone inlet angles on the design and can describe the collection efficiency of the sampler. Experimental data for the cyclone sampler with different inlet angles show a minimum collection efficiency of about $82 \%$. Particle diameter from $0.5 \mu \mathrm{m}$ to $16 \mu \mathrm{m}$ for the polymer microspheres were used in sample analysis on a cyclone based bioaerosol sampler. table 5.1 shows a remarkable trend in terms of particle collection efficiency on performance parameter. The value for the smallest inlet angle $\left(30^{\circ}\right)$ does not show a very large-scale change with the $40^{\circ}$ inlet section. Particle collection efficiency is increased from $83.51 \%$ for the $30^{\circ}$ inlet angle to $95.58 \%$ when the $40^{\circ}$ angle is used. The result obtained in this study is $99.94 \%$ for $45^{\circ}$ inlet tube angle. Conversely, the collection efficiency for the $60^{\circ}$ angle is less than that for the $45^{\circ}$ angle.

From figure 5.15, it can be inferred that more particle retained in the microcentrifuge tube with the increase of particle size. So, if the micron-particle is large in size, higher collection efficiency of the cyclone sampler can be obtained. In this study, the desired angle is positioned most probably close to $45^{\circ}$ inlet section. Among these four different types of cyclone sampler, $45^{\circ}$ inlet tube angle showed more particle collection efficiency compared to other bioaerosol cyclone sampler. The interim value between these inlet tubes angles indicate that $45^{\circ}$ inlet tube would be an ideal angle for this study in which the best collection efficiency results can be 
obtained. Therefore, it can be recommended from this study that changing scroll inlet angle is an expedient way to improve cyclone sampler performance. 


\section{Chapter 6: Conclusion}

We applied 3D full order high-fidelity CFD tool simulate particle motion and particle-air interaction in a personal bio-aerosol sampler. Four cyclone sampler of different inlet tube angle have been simulated using the Reynolds stress model (RSM), to observe the effect on cyclone performance and flow pattern. FLUENT 13.0 were used to accomplish the Computational Fluid Dynamics analysis. The inlet angles of the cyclone sampler have an impact on particle entrance which affects cyclone efficiency in a statistically effective way. Changing the inlet tube angle makes a considerable change in cyclone sampler performances. This study recommends a new personal cyclone sampler design by using $30^{\circ}, 40^{\circ}, 45^{\circ}$ and $60^{\circ}$ angles for the inlet section. Several fluid dynamics profiles and performance parameter were investigated, presented and discussed. The fluid flow of the cyclone sampler has been characterized with different inlet angle and flow velocity with corresponding velocity fluctuation profiles of axial, radial and tangential components, which highlight the influence of wall boundary later on particle deposits on the collection tube. The RSM exhibits a significant representation of fluid flow and fluid-particle turbulent swirling flow behavior in cyclone sampler with the help of CFD technique. In order to comprehend the sampler inlet tube angle effects on its collection efficiency based on the fluid dynamics phenomena, elaborative flow field description was achieved through CFD simulation. New characteristics of velocity distribution and particle tracks were obtained by application of the RSM and DPM. Performance parameter such as collection efficiency indicates that this different inlet angle application can give an alternative way to improve sampler performance.

Numerical results show that the inlet angle of $45^{\circ}$ leads to a better collection efficiency which is $99.94 \%$ where the inlet velocity and collection tube surface roughness were kept same for different inlet tube angle. For the same inlet angle, the roughest inner wall leads to the best 
collection efficiency. We have not done with the numerical analysis of two-stage cyclone microcentrifuge tube sampler for the size segregated sample collection yet. We have plans in future to perform numerical simulation on a two-stage cyclone sampler to observe the particle collection ability of the sampler by using the current CFD techniques. 


\section{References}

1. Chen, B.T., et al., Development of a personal sampler for collecting fungal spores. Aerosol science and technology, 2004. 38(9): p. 926-937.

2. $\quad$ Lindsley, W.G., D. Schmechel, and B.T. Chen, A two-stage cyclone using microcentrifuge tubes for personal bioaerosol sampling. Journal of Environmental Monitoring, 2006. 8(11): p. 1136-1142.

3. Redrow, J., et al., Modeling the evaporation and dispersion of airborne sputum droplets expelled from a human cough. Building and Environment, 2011. 46(10): p. 2042-2051.

4. Shukla, S.K., P. Shukla, and P. Ghosh, Evaluation of numerical schemes for dispersed phase modeling of cyclone separators. Engineering Applications of Computational Fluid Mechanics, 2011. 5(2): p. 235-246.

5. Bernardo, S., et al., 3-D computational fluid dynamics for gas and gas-particle flows in a cyclone with different inlet section angles. Powder Technology, 2006. 162(3): p. 190-200.

6. $\quad$ Ksiazek, T.G., et al., A novel coronavirus associated with severe acute respiratory syndrome. New England Journal of Medicine, 2003. 348(20): p. 1953-1966.

7. Kulkarni, P., P.A. Baron, and K. Willeke, Aerosol measurement: principles, techniques, and applications. 2011: John Wiley \& Sons.

8. Hinds, W.C., Aerosol technology: properties, behavior, and measurement of airborne particles. 2012: John Wiley \& Sons.

9. Cox, C.S. and C.M. Wathes, Bioaerosols handbook. 1995: crc press.

10. Marple, V.A., K.L. Rubow, and S.M. Behm, A microorifice uniform deposit impactor (MOUDI): Description, calibration, and use. Aerosol Science and Technology, 1991. 14(4): p. 434-446.

11. Marple, V.A. and B.Y. Liu, On fluid flow and aerosol impaction in inertial impactors. Journal of Colloid and Interface Science, 1975. 53(1): p. 31-34.

12. McMurry, P.H., A review of atmospheric aerosol measurements. Atmospheric Environment, 2000. 34(12): p. 1959-1999.

13. Lin, X., et al., Effect of sampling time on the collection efficiency of all-glass impingers. American Industrial Hygiene Association Journal, 1997. 58(7): p. 480-488.

14. Ghosh, T.K. and M.A. Prelas, Sensors and Detection Systems for Biological Agents. Science and Technology of Terrorism and Counterterrorism, 2002.

15. Hering, S.V., Impactors, cyclones, and other inertial and gravitational collectors. Air sampling instruments for evaluation of atmospheric contaminants. 8th ed. Cohen and Hering, editors. Cincinnati (OH): ACGIH, 1995.

16. Flocchini, R., et al., Characterization of particles in the arid west. Atmospheric Environment (1967), 1981. 15(10): p. 2017-2030.

17. Griffiths, W. and I. Stewart, Performance of bioaerosol samplers used by the UK biotechnology industry. Journal of aerosol science, 1999. 30(8): p. 1029-1040.

18. Griffiths, W. and F. Boysan, Computational fluid dynamics (CFD) and empirical modelling of the performance of a number of cyclone samplers. Journal of Aerosol Science, 1996. 27(2): p. 281-304.

19. Delfino, R.J., et al., The effect of outdoor fungal spore concentrations on daily asthma severity. Environmental health perspectives, 1997. 105(6): p. 622. 
20. Li, D.-W., Stachybotrys eucylindrospora, sp. nov. resulting from a re-examination of Stachybotrys cylindrospora. Mycologia, 2007. 99(2): p. 332-339.

21. Zhou, G., et al., Development of a fungus-specific PCR assay for detecting low-level fungi in an indoor environment. Molecular and cellular probes, 2000. 14(6): p. 339-348.

22. Cruz-Perez, P., M. Buttner, and L. Stetzenbach, Detection and quantitation of $<i>$ Aspergillus fumigatus $</ i>$ in pure culture using polymerase chain reaction. Molecular and cellular probes, 2001. 15(2): p. 81-88.

23. Schmechel, D., et al., Limitations of monoclonal antibodies for monitoring of fungal aerosols using $<i>$ Penicillium brevicompactum $</ i>$ as a model fungus. Journal of immunological methods, 2003. 283(1): p. 235-245.

24. Ladeinde, F. and M.D. Nearon, CFD applications in the HVAC\&R industry. ASHRAE journal, 1997. 39: p. 44-48.

25. Hirsch, C., Numerical Computation of Internal and External Flows: The Fundamentals of Computational Fluid Dynamics: The Fundamentals of Computational Fluid Dynamics. Vol. 1. 2007: Butterworth-Heinemann.

26. Chu, K., et al., CFD-DEM simulation of the gas-solid flow in a cyclone separator. Chemical Engineering Science, 2011. 66(5): p. 834-847.

27. $\mathrm{Chu}, \mathrm{K}$. and $\mathrm{A}$. Yu, Numerical simulation of complex particle-fluid flows. Powder Technology, 2008. 179(3): p. 104-114.

28. Tsuji, Y., T. Tanaka, and T. Ishida, Lagrangian numerical simulation of plug flow of cohesionless particles in a horizontal pipe. Powder technology, 1992. 71(3): p. 239-250.

29. $\mathrm{Xu}, \mathrm{B}$. and $\mathrm{A}$. Yu, Numerical simulation of the gas-solid flow in a fluidized bed by combining discrete particle method with computational fluid dynamics. Chemical Engineering Science, 1997. 52(16): p. 2785-2809.

30. Li, Y., J. Zhang, and L.-S. Fan, Numerical simulation of gas-liquid-solid fluidization systems using a combined CFD-VOF-DPM method: bubble wake behavior. Chemical Engineering Science, 1999. 54(21): p. 5101-5107.

31. Rhodes, M., et al., Use of discrete element method simulation in studying fluidization characteristics: influence of interparticle force. Chemical Engineering Science, 2001. 56(1): p. 69-76.

32. Kafui, K., C. Thornton, and M. Adams, Discrete particle-continuum fluid modelling of gas-solid fluidised beds. Chemical Engineering Science, 2002. 57(13): p. 2395-2410.

33. Yu, A.B. and B.H. Xu, Particle-scale modelling of gas-solid flow in fluidisation. Journal of Chemical Technology and Biotechnology, 2003. 78(2-3): p. 111-121.

34. Limtrakul, S., A. Boonsrirat, and T. Vatanatham, DEM modeling and simulation of a catalytic gas-solid fluidized bed reactor: a spouted bed as a case study. Chemical Engineering Science, 2004. 59(22): p. 5225-5231.

35. Di Renzo, A. and F.P. Di Maio, Homogeneous and bubbling fluidization regimes in DEM-CFD simulations: hydrodynamic stability of gas and liquid fluidized beds. Chemical Engineering Science, 2007. 62(1): p. 116-130.

36. Tsuji, Y., Multi-scale modeling of dense phase gas-particle flow. Chemical Engineering Science, 2007. 62(13): p. 3410-3418.

37. Malone, K. and B. Xu, Particle-scale simulation of heat transfer in liquid-fluidised beds. Powder Technology, 2008. 184(2): p. 189-204.

38. Kawaguchi, T., T. Tanaka, and Y. Tsuji, Numerical simulation of two-dimensional fluidized beds using the discrete element method (comparison between the two-and threedimensional models). Powder technology, 1998. 96(2): p. 129-138. 
39. Ibsen, C.H., et al., Comparison of multifluid and discrete particle modelling in numerical predictions of gas particle flow in circulating fluidised beds. Powder Technology, 2004. 149(1): p. 29-41.

40. Tryggvason, G., et al., A front-tracking method for the computations of multiphase flow. Journal of Computational Physics, 2001. 169(2): p. 708-759.

41. Chu, K., et al., CFD-DEM study of the effect of particle density distribution on the multiphase flow and performance of dense medium cyclone. Minerals Engineering, 2009. 22(11): p. 893-909.

42. Chu, K., et al., CFD-DEM modelling of multiphase flow in dense medium cyclones. Powder Technology, 2009. 193(3): p. 235-247.

43. Liu, D., C. Bu, and X. Chen, Development and test of CFD-DEM model for complex geometry: A coupling algorithm for Fluent and DEM. Computers \& Chemical Engineering, 2013. 58: p. 260-268.

44. Batchelor, G.K., An introduction to fluid dynamics. 2000: Cambridge university press.

45. Peyret, R. and T.D. Taylor, Computational methods for fluid flow. New York, SpringerVerlag, 1985, 368 p., 1985. 1.

46. Hoffmann, A.C. and L.E. Stein, Gas Cyclones and Swirl Tubes: Principles, Design, and Operation. 2007: Springer.

47. Fluent-Inc, "Fluent 6.3. User's Guide,". 2006, Fluent Inc.

48. Crowe, C.T., et al., Multiphase flows with droplets and particles. 2011: CRC press.

49. Krist, S.L., R.T. Biedron, and C.L. Rumsey, CFL3D user's manual (version 5.0). 1998: Citeseer.

50. Gimbun, J., et al., A CFD study on the prediction of cyclone collection efficiency. International Journal for Computational Methods in Engineering Science and Mechanics, 2005. 6(3): p. 161-168.

51. Shaw, B.W., Analysis of Cyclone Collection Efficiency. 2003.

52. Dietz, P., Collection efficiency of cyclone separators. AIChE Journal, 1981. 27(6): p. 888-892.

53. Elsayed, K. and C. Lacor, A CFD Study of the Effects of Cone Dimensions on the Flow Field of Cyclone Separators using LES.

54. Boysan, F., W. Ayers, and J. Swithenbank, A fundamental mathematical modelling approach to cyclone design. Transactions of the Institution of Chemical Engineers, 1982. 60(4): p. 222-230.

55. Hoekstra, A., J. Derksen, and H. Van Den Akker, An experimental and numerical study of turbulent swirling flow in gas cyclones. Chemical Engineering Science, 1999. 54(13): p. 2055-2065.

56. Pant, K., C. Crowe, and P. Irving, On the design of miniature cyclones for the collection of bioaerosols. Powder Technology, 2002. 125(2): p. 260-265.

57. Sommerfeld, M. and C.A. Ho, Numerical calculation of particle transport in turbulent wall bounded flows. Powder Technology, 2003. 131(1): p. 1-6.

58. Jayaraju, S.T., STUDY OF THE AIR FLOW AND AEROSOL TRANSPORT IN THE HUMAN UPPER AIRWAY USING LES AND DES METHODOLOGIES. Phd diss., Vrije Universiteit Brussel, Brussels, Belgium, 2009.

59. Meier, H.F. and M. Mori, Anisotropic behavior of the Reynolds stress in gas and gassolid flows in cyclones. Powder Technology, 1999. 101(2): p. 108-119.

60. Bernardo, S., A. Peres, and M. Mori, Computational study of cyclone flow fluid dynamics using a different inlet section angle. Revista de Engenharia Térmica, 2005. 4(1). 
61. de Nunes Fabião, P., V.R. Wiggers, and E.L. Reinehr, ANÁLISE EXPERIMENTAL E NUMÉRICA DO ESCOAMENTO MULTIFÁSICO GÁS-SÓLIDO EM CICLONES. 2010. 


\section{Appendix}

\section{Nomenclature}

$\begin{array}{ll}\nabla & \text { Gradient operator } \\ \boldsymbol{C}_{\boldsymbol{d}} & \text { Drag force coefficient } \\ \boldsymbol{d}_{\boldsymbol{i j}} & \text { Deformation tensor } \\ \boldsymbol{d}_{\boldsymbol{p}} & \text { Particle diameter } \\ \boldsymbol{F}_{\boldsymbol{F}} & \text { Saffman's lift force } \\ \boldsymbol{F}_{\boldsymbol{D}} & \text { Drag force } \\ \boldsymbol{F}_{\boldsymbol{x}} & \text { additional acceleration }\left(\mathrm{m} / \mathrm{s}^{2}\right) \\ \boldsymbol{g}_{\boldsymbol{x}} & \text { gravitational acceleration }\left(\mathrm{m} / \mathrm{s}^{2}\right) \\ \boldsymbol{k} & \text { turbulent kinetic energy per unit mass }\left(\mathrm{m}^{2} / \mathrm{s}^{2}\right) \\ \boldsymbol{P}_{\boldsymbol{i j}} & \text { production term } \\ \boldsymbol{R} \boldsymbol{e} & \text { Reynolds number } \\ \boldsymbol{t} & \text { time } \\ \boldsymbol{u} & \text { flow velocity (m/s) } \\ \boldsymbol{u}_{p} & \\ & \end{array}$

\section{Greek symbols}

$\varepsilon \quad$ Dissipation rate of turbulent kinetic energy

$\rho \quad$ Fluid density

$\mu \quad$ Viscosity 
$\rho_{\mathrm{p}} \quad$ particle density

\title{
Subscripts
}

$\mathrm{i}, \mathrm{j}, \mathrm{k}$ unit vectors in $\mathrm{x}, \mathrm{y}$ and $\mathrm{z}$ directions

p particle

d drag

\author{
Abbreviations \\ BC boundary condition \\ CFD computational fluid dynamics \\ DPM discrete phase model \\ DRW discrete random walk \\ FVM finite volume method
}

RSTM Reynolds stress turbulence modelling

SIMPLEC Semi-Implicit Method for Pressure-Linked Equations-Consistent 


\section{Curriculum Vita}

Antara Badhan, only one child of Md. Abdur Rahman Mia and Nurzahan Begum, was born on $21^{\text {st }}$ September, 1989 in Dhaka, Bangladesh. Ms. Badhan completed her secondary school certificate from Monipur High School and higher secondary school certificate from Dhaka City College, Bangladesh and then joined Khulna University of Engineering \& Technology (KUET) for Bachelor of Science in Civil Engineering in February, 2007. She has completed her Bachelor degree in May 2011 from KUET. She gained knowledge on Engineering Measurements (Land Surveying), Foundation engineering and Hydraulic Engineering during her undergraduate course. Ms. Badhan joined the University of Texas at El Paso (UTEP) to pursue Master of Science degree in Mechanical Engineering in January 2013. She has been working as a Graduate Research Assistant in Computational Fluid Mechanics Lab. Her work was focused on numerical analysis of particle flow inside a bio-aerosol cyclone sampler to evaluate particle collection efficiency for different design options. . In addition to her research work, Ms. Badhan served teaching assistant duties in last two semesters in undergraduate level Thermal System Design. She has instructed ungraduated students on their experiments/labs session in Lockheed Martin Mechanical Engineering Lab at UTEP. She is a coauthor of 2 conference papers to be published this year and is currently working on a journal draft as well as other proposals.

Permanent Address: 73/a, Kadamtala, Bashaboo, Sabujbagh

Dhaka, Bangladesh.

Author's Name: Antara Badhan 